\title{
Modeling Approaches to Assess Soil Erosion by Water at the Field Scale with Special Emphasis on Heterogeneity of Soils and Crops
}

\author{
Ahsan Raza ${ }^{1, *}$, Hella Ahrends ${ }^{1,2}$, Muhammad Habib-Ur-Rahman ${ }^{1,3} \mathbb{D}$ and Thomas Gaiser 1 \\ 1 Institute of Crop Science and Resource Conservation (INRES), Crop Science Group, University of Bonn, \\ Katzenburgweg 5, 53115 Bonn, Germany; ellen.ahrends@helsinki.fi (H.A.); mhabibur@uni-bonn.de or \\ habib.rahman@mnsuam.edu.pk (M.H.-U.-R.); tgaiser@uni-bonn.de (T.G.) \\ 2 Department of Agricultural Sciences, University of Helsinki, 00014 Helsinki, Finland \\ 3 Department of Agronomy, MNS-University of Agriculture, Multan, Punjab 60000, Pakistan \\ * Correspondence: araza@uni-bonn.de; Tel.: +49-0-228-73-7200
}

Citation: Raza, A.; Ahrends, H.; Habib-Ur-Rahman, M.; Gaiser, T. Modeling Approaches to Assess Soil Erosion by Water at the Field Scale with Special Emphasis on Heterogeneity of Soils and Crops. Land 2021, 10, 422. https://doi.org/ 10.3390/land10040422

Academic Editor: Deirdre Dragovich

Received: 16 March 2021

Accepted: 6 April 2021

Published: 15 April 2021

Publisher's Note: MDPI stays neutra with regard to jurisdictional claims in published maps and institutional affiliations.

Copyright: (c) 2021 by the authors. Licensee MDPI, Basel, Switzerland. This article is an open access article distributed under the terms and conditions of the Creative Commons Attribution (CC BY) license (https:/ / creativecommons.org/licenses/by/ $4.0 /)$.

\begin{abstract}
Information on soil erosion and related sedimentation processes are very important for natural resource management and sustainable farming. Plenty of models are available for studying soil erosion but only a few are suitable for dynamic soil erosion assessments at the field-scale. To date, there are no field-scale dynamic models available considering complex agricultural systems for the simulation of soil erosion. We conducted a review of 51 different models evaluated based on their representation of the processes of soil erosion by water. Secondly, we consider their suitability for assessing soil erosion for more complex field designs, such as patch cropping, strip cropping and agroforestry (alley-cropping systems) and other land management practices. Several models allow daily soil erosion assessments at the sub-field scale, such as EPIC, PERFECT, GUEST, EPM, TCRP, SLEMSA, APSIM, RillGrow, WaNuLCAS, SCUAF, and CREAMS. However, further model development is needed with respect to the interaction of components, i.e., rainfall intensity, overland flow, crop cover, and their scaling limitations. A particular shortcoming of most of the existing field scale models is their one-dimensional nature. We further suggest that platforms with modular structure, such as SIMPLACE and APSIM, offer the possibility to integrate soil erosion as a separate module/component and link to GIS capabilities, and are more flexible to simulate fluxes of matter in the 2D/3D dimensions. Since models operating at daily scales often do not consider a horizontal transfer of matter, such modeling platforms can link erosion components with other environmental components to provide robust estimations of the three-dimensional fluxes and sedimentation processes occurring during soil erosion events.
\end{abstract}

Keywords: erosion and sedimentation processes; model categorization; complex cropping systems; governing equations; application

\section{Introduction}

Soil erosion is a significant problem worldwide for most of the agro-ecosystems [1] because it is one of the primary causes of soil degradation as a result of detachment and loss of topsoil layer and soil organic matter, which are essential for plant development. Quantification of soil loss related to soil and crop management, climate, and soil conditions has, therefore, become a serious concern for water and soil conservation practitioners, as well as decision-makers concerned with food security and agricultural policies [2]. Soil erosion is the process of detachment and transportation of soil particles involving various erosive agents from the earth's surface. Categorized into wind and water erosion, water erosion is a much more complex process and leads to substantial loss of soil and sedimentation [3]. Water erosion is mainly affected by rainfall/runoff intensity, vegetation cover, soil erodibility, topography, and land use management practices [4]. 
Due to the rapid advancement in data computing techniques in the last three decades, there is a substantial enhancement in the analysis of soil erosion through the development of computer models [5]. However, these models strongly differ in terms of data requirement, application scales, and complexity, along with uncertainties in the individual factors of the respective models [6]. Water erosion modeling is about 60 years old, but has become a key factor in our understanding of the complexity of erosion processes and for predicting future scenarios. Yet, most of the models are still inadequate due to multiple sources of uncertainty $[7,8]$.

Many different algorithms and relations have been proposed to define and predict soil erosion by water and associated sediment yield, varying noticeably in their objectives, time scale at the plot level, and in their conceptual basis as well. The choice of the most suitable model is a logical process affected by many factors including land use, the characteristics of the catchment being considered, and the data available [9]. Physically based models, for example, mainly depend on the principal approach of mass and energy conservation to simulate runoff and sedimentation. In addition, physically based models are based on the concept of physics using transfer of momentum as a governing equation [10].

Remote Sensing and GIS have huge potential for analysis and mapping of parameters influencing soil erosion and degraded lands in quantitative and qualitative manners. However, the use of GIS for soil erosion modeling requires facilitations such as multiple data resources, data scaling, and increased complexity in data integration and algorithms. Climate, land use/land cover, topography, and slope data can be assessed using LIDAR or Satellite imageries and can be integrated with GIS for soil erosion, transport, and sedimentation modeling [11-14].

There is an increasing interest in more complex field designs and crop diversification on the same field, e.g. alley cropping systems, as one of the most popular type of agroforestry, patch cropping, strip cropping, and uncultivated drylands, wherein rotational grazing livestock are moved to a part of the pasture, while the other portions rest, which can impact modeling outcomes. Consequently, a need for suitable soil erosion models that can handle and consider more complex field designs is raised.

The key objective of this study is, therefore, to categorize an extensive amount of available soil erosion models, review the underlying concepts, data requirements, and sources of uncertainty. We especially consider their suitability to simulate soil erosion at the sub-field scale and their application for more complex field designs. More specifically, we aim to

i. Provide a review of a large number of existing soil erosion models with respect to

(a) the challenges for simulating field-scale erosion processes and (b) consideration of more complex cropping systems like alley cropping, patch cropping, and strip cropping, and based on these findings,

ii. Provide suggestions on a way forward for corresponding model improvements.

\section{Materials and Methods}

We performed a systematic model review with the reviewed soil erosion models being based on the outcome of a thorough literature screening, identification of suitable models, and model classification (Figure 1). This review paper is structured in the following way. A brief explanation of soil erosion, transportation, and sedimentation principles is made in Section 3.1. In this review, more than 60 models were reviewed and 51 models were retained in Section 3.2. The shortlisted models are reviewed in terms of their objective, model structure, model components, as well as their application, ease of model calibration, and parameter requirements. Models are categorized in terms of their ability to explain the soil erosion processes, governing equations, their spatial and temporal resolution, their capabilities, and their limitations. These models have a wide range of applications from point scale to catchment scale. The focus of this review primarily considers models having abilities to simulate soil erosion process at the field scale and in complex cropping systems. The selected models are described in Section 3.3. in which it is noted that many 
field scale models are implemented for catchment-scale soil erosion simulations. From a wider point of view, some of these models are described under their respective categories. In Section 4, a discussion summarizes descriptions of models to sort out which model fits which conditions and problems identified and leads to clear guidelines to select the appropriate model. This discussion is used to identify key points that would enhance the quality of the modeling output and the nature of additional components to enhance model capability in most environmental and management conditions. Section 5 provides a way forward on how to improve and extend existing models to simulate erosion processes at a small spatial scale in complex agriculture systems.

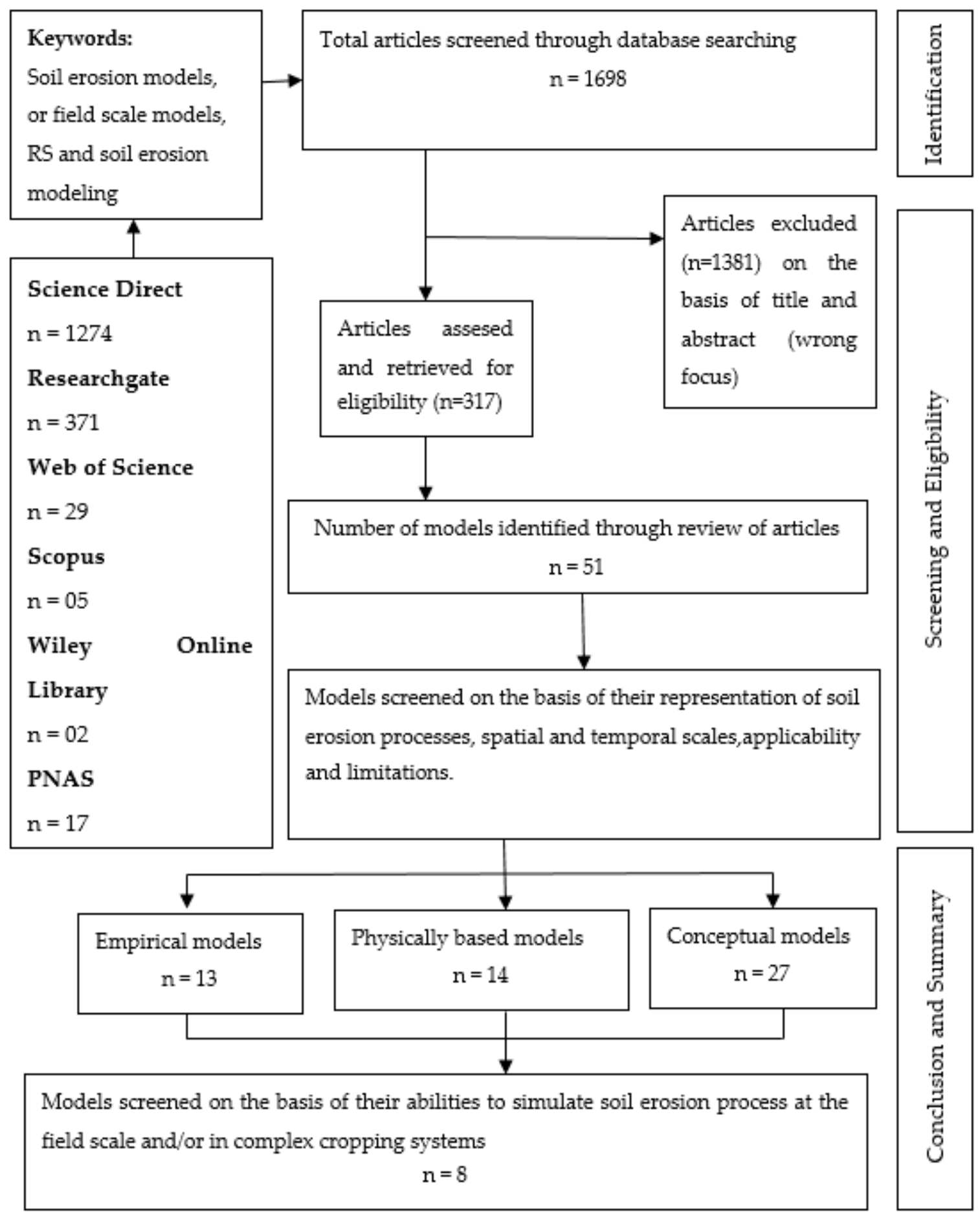

Figure 1. The literature study flow diagram. 


\section{Results}

\subsection{Principle for Erosion Modelling}

Sedimentological and hydrological processes involved in the modeling of soil erosion by water are explained mainly by two principles representing these processes (Table 1). Every erosion model can be considered as a unique permutation of these two principles [15].

Table 1. Principles driving process representation in soil erosion models (modified after [15]).

\begin{tabular}{lr}
\hline \multicolumn{1}{c}{ Principle } & Summary \\
\hline $\begin{array}{l}\text { A model must represent all factors significantly contributing to the } \\
\text { erosion process at the spatial, temporal, and locality levels for which the } \\
\text { model is applied. }\end{array}$ & What to represent \\
\hline $\begin{array}{l}\text { A model may apply different weights to the individual processes or it } \\
\text { may represent these processes directly, indirectly, or using a hybrid } \\
\text { approach. [16]. }\end{array}$ & How to represent \\
\hline
\end{tabular}

One of the critical aspects of the first principle (Table 1) is that every erosion model operates at different temporal and spatial scales [17]. Therefore, a plot-scale model must be able to represent a different combination of erosion processes as compared to those developed for the landscape (i.e., watershed or regional) scale (Table 2). Further, if simulating single events, the processes represented in the model may differ from those considered in models for long-term simulations or the weighting factors for each of the processes may be different. Similarly, erosion processes vary depending on the climatic conditions (i.e., humid, arid, etc.) and models developed for these specific regions must vary in terms of the number and type of erosion processes that are considered [16].

Table 2. Spatial scale sizes for soil erosion modeling.

\begin{tabular}{ccc}
\hline Category & Spatial Scale & Size \\
\hline \multirow{2}{*}{ Large scale } & Basin & $>500 \mathrm{~km}^{2}$ \\
\cline { 2 - 3 } & Catchment & $50-500 \mathrm{~km}^{2}$ \\
\cline { 2 - 3 } & Watershed & $1-50 \mathrm{~km}^{2}$ \\
\hline \multirow{2}{*}{ Small scale } & Field/hillslope & $<1 \mathrm{~km}^{2}$ \\
\cline { 2 - 3 } & Plot & $0.6-23 \mathrm{~m}^{2}$ \\
\hline
\end{tabular}

\subsection{Soil Detachment and Sedimentation Assessment Model Approaches}

A wide range of modeling approaches has been developed for simulating soil erosion and sedimentation over the last decades, differing in their representation of processes involved in soil erosion, the complexity of these processes, data requirements and output uncertainties, model calibration and use, and their temporal and spatial scale limitations. In general, the model selection depends on the intended application and characteristics of the landscape. Therefore, several factors must be considered before the model selection i.e., objective, data requirements, data availability, accuracy, validity, etc.

Each model has been designed for a specific spatial scale and purpose and thus is not appropriate and suitable for every application. Based on the complexity and the level of dynamic physical processes that are implemented, models can be categorized into three different groups, namely empirical, conceptual, and physically based models. Due to the increasing application of geospatial data, we further distinguish a fourth category: Remote Sensing and GIS-based modeling approaches. However, most of the models might be composed of different model categories. For example, the runoff-rainfall component of the USLE model [18] may be physically based but an empirical relationship has been developed for the estimation of soil erosion and sediment yield with little computational efforts. An example of so-called "hybrid models" is the Unit Stream Power-based Erosion 
Deposition and Automated Geospatial Watershed Assessment. The model structure is conceptual in nature considering the number of storages, while the configuration of these storages is determined through a statistical identification process for each catchment. The accuracy of these models is mainly dependent on the parameters selected and their primary implications. Alewell et al. [19] noted the primarily different nature of gross (modeled) vs net (measured) soil erosion.

\subsubsection{Empirical Models}

Empirical models are primarily based on observation data and the relationships between different factors and soil erosion levels that were derived from these data sets. The computational and input data requirements for empirical models are lower than those required for conceptual or physically based models. Hence, empirical models are comparatively flexible, have a simple structure, are easily implemented, and useful in identifying the source of sedimentation generation as a first step. The most critical limitation of empirical models for soil erosion is their inadequate level of accuracy in analyzing large data sets which would require processing and analysis using special complex mathematical approaches [20].

Empirical models have proven to be robust since they are mathematically simple, but their application is limited to the extent of area for which they have been developed and calibrated for the fact that users will not get benefit from complex models if incomplete input data is available. They are often based on standard runoff plot schemes for uniform slopes [21]. At regional scales, with the identification of sediment settlement and delivery patterns, empirical models can be applied to predict average sedimentation, soil erosion rates, and surface runoff using the SCS curve number. If soil characteristics spatially do not vary and if spatially explicit meteorological data is not available, the application of robust empirical models can provide more reliable results as compared with more complex and dynamic models. However, empirical models work on the concept of stationarity, which makes them less powerful for predicting soil erosion for complex terrains characterized by heterogeneous soil characteristics and climatic conditions. Hence, empirical models are often applied when the availability of model input data is limited. Most of the empirical models do not provide information regarding sediment deposition and stream sedimentation generation, which restricts their application for simulating mass balances.

There are a few field-scale models such as EPM, TCRP, and SLEMSA which simulate soil detachment, transportation, and sedimentation using predominantly empirical approaches at field-scale (Table 3). The PSIAC model has the ability to estimate soil erosion and sedimentation at both field and catchment scales. These models are continuous simulation models that are useful for predicting the effects of field management practices and the effects of hydrological variations at daily time steps (Table 3 ). There are a few empirical models available to study the soil erosion processes under agroforestry systems such as WaNuLCAS, SCUAF, and HyPAR. Most of the models contain process-based sub-models to simulate the crop growth based on their vegetative and generative stages under specific field conditions [22] including all soil processes that may affect agricultural systems, such as C, P, N dynamics, and soil erosion [22]. Models such as USLE, MUSLE, RUSLE, and MOSES have long-term simulation capabilities at both hillslope and catchment scale. Models are distinguished on the basis of spatial and temporal scale as detailed in Table 3. 
Table 3. Empirical soil erosion models.

\begin{tabular}{|c|c|c|c|c|c|c|c|c|c|c|c|c|c|c|c|}
\hline \multirow{2}{*}{ Sr. No. } & \multirow{2}{*}{ Model } & \multirow{2}{*}{ Description } & \multirow{2}{*}{$\begin{array}{l}\text { Developer } \\
\text { /Year* }\end{array}$} & \multicolumn{2}{|c|}{ Scale } & \multicolumn{2}{|c|}{ Input } & \multirow{2}{*}{$\begin{array}{l}\text { Governing } \\
\text { Equations }\end{array}$} & \multirow{2}{*}{$\begin{array}{l}\text { Model } \\
\text { Capabilities }\end{array}$} & \multirow{2}{*}{$\begin{array}{c}\text { Model } \\
\text { Limitations }\end{array}$} & \multicolumn{3}{|c|}{$\begin{array}{c}\text { Overland } \\
\text { Sedimentation }\end{array}$} & \multirow{2}{*}{$\begin{array}{c}\text { Channel } \\
\text { Sedimentation } \\
\text { Generation }\end{array}$} & \multirow{2}{*}{ Source } \\
\hline & & & & Temporal & Spatial & Demand & Variables & & & & $\mathrm{G}^{*}$ & $\mathrm{~T}^{*}$ & $\mathbf{D}^{*}$ & & \\
\hline 1 & USLE & $\begin{array}{l}\text { Universal Soil } \\
\text { Loss } \\
\text { Equation }\end{array}$ & [23] & Annual & $\begin{array}{l}\text { Catchment/ } \\
\text { Hillslope }\end{array}$ & High & $\begin{array}{l}\text { Climate data, } \\
\text { topography, } \\
\text { Land use/Land } \\
\text { cover, field } \\
\text { management } \\
\text { practices, crop } \\
\text { management } \\
\text { factor }\end{array}$ & $\begin{array}{l}\text { Universal } \\
\text { Soil Loss } \\
\text { Equation }\end{array}$ & Erosion & $\begin{array}{l}\text { Does not } \\
\text { quantify the } \\
\text { events that are } \\
\text { likely to result } \\
\text { in large-scale } \\
\text { erosion }\end{array}$ & No & Yes & No & No & [24] \\
\hline 4 & MOSES & $\begin{array}{l}\text { Modular Soil } \\
\text { Erosion } \\
\text { System } \\
\text { project }\end{array}$ & [29] & Annual & $\begin{array}{l}\text { Catchment/ } \\
\text { Hillslope }\end{array}$ & High & $\begin{array}{l}\text { Climate data, } \\
\text { topography, } \\
\text { Land use/Land } \\
\text { cover, } \\
\text { field } \\
\text { management } \\
\text { practices, crop } \\
\text { management } \\
\text { factor }\end{array}$ & $\begin{array}{l}\text { Enhanced } \\
\text { Revised } \\
\text { Universal Soil } \\
\text { Loss } \\
\text { Equation } \\
\text { (RUSLE2), } \\
\text { Wind Erosion } \\
\text { Prediction } \\
\text { System } \\
\text { (WEPS) } \\
\text { model }\end{array}$ & $\begin{array}{l}\text { Wind erosion, } \\
\text { water erosion } \\
\text { sediment } \\
\text { yield, } \\
\text { runoff }\end{array}$ & $\begin{array}{l}\text { Does not } \\
\text { consider gully } \\
\text { erosion }\end{array}$ & No & Yes & No & No & [29] \\
\hline
\end{tabular}


Table 3. Cont

\begin{tabular}{|c|c|c|c|c|c|c|c|c|c|c|c|c|c|c|c|}
\hline \multirow{2}{*}{ Sr. No. } & \multirow{2}{*}{ Model } & \multirow{2}{*}{ Description } & \multirow{2}{*}{$\begin{array}{c}\text { Developer } \\
\text { /Year* }\end{array}$} & \multicolumn{2}{|c|}{ Scale } & \multicolumn{2}{|c|}{ Input } & \multirow{2}{*}{$\begin{array}{l}\text { Governing } \\
\text { Equations }\end{array}$} & \multirow{2}{*}{$\begin{array}{l}\text { Model } \\
\text { Capabilities }\end{array}$} & \multirow{2}{*}{$\begin{array}{c}\text { Model } \\
\text { Limitations }\end{array}$} & \multicolumn{3}{|c|}{$\begin{array}{c}\text { Overland } \\
\text { Sedimentation }\end{array}$} & \multirow{2}{*}{$\begin{array}{c}\text { Channel } \\
\text { Sedimentation } \\
\text { Generation }\end{array}$} & \multirow{2}{*}{ Source } \\
\hline & & & & Temporal & Spatial & Demand & Variables & & & & $\mathrm{G}^{*}$ & $\mathrm{~T}^{*}$ & $\mathrm{D}^{*}$ & & \\
\hline 5 & SEDD & $\begin{array}{l}\text { Sediment } \\
\text { Delivery } \\
\text { Distributed }\end{array}$ & [30] & Annual & $\begin{array}{l}\text { Basin, large } \\
\text { catchment }\end{array}$ & High & $\begin{array}{l}\text { DEM, a land } \\
\text { use } \\
\text { Map, climate, } \\
\text { human } \\
\text { influence }\end{array}$ & $\begin{array}{l}\text { Universal Soil } \\
\text { Loss } \\
\text { Equation }\end{array}$ & $\begin{array}{l}\text { Basin } \\
\text { sediment } \\
\text { yields }\end{array}$ & $\begin{array}{c}\text { Model } \\
\text { reliability } \\
\text { decreases from } \\
\text { the annual scale } \\
\text { to the event } \\
\text { scale }\end{array}$ & No & Yes & No & No & [30] \\
\hline 6 & EPM & $\begin{array}{l}\text { Erosion } \\
\text { Potential } \\
\text { Method }\end{array}$ & [31] & Annual & Field & High & $\begin{array}{l}\text { Climate data, } \\
\text { topography, } \\
\text { area of } \\
\text { catchment, } \\
\text { stream network, } \\
\text { soil erodibility } \\
\text { coefficient }\end{array}$ & $\begin{array}{l}\text { Analytical } \\
\text { equation for } \\
\text { spatial and } \\
\text { temporal } \\
\text { variation } \\
\text { measurement }\end{array}$ & $\begin{array}{l}\text { Retention } \\
\text { coefficient } \\
\text { Erosion } \\
\text { intensity, } \\
\text { sediment } \\
\text { production, } \\
\text { sediment } \\
\text { transport }\end{array}$ & $\begin{array}{l}\text { Performance } \\
\text { subjected to the } \\
\text { specific } \\
\text { characteristics } \\
\text { and } \\
\text { sedimentary } \\
\text { regime of the } \\
\text { study area }\end{array}$ & No & Yes & No & No & [32] \\
\hline 7 & TCRP & $\begin{array}{l}\text { Tillage- } \\
\text { Controlled } \\
\text { Runoff } \\
\text { Pattern } \\
\text { model }\end{array}$ & [33] & $\begin{array}{l}\text { Event/ } \\
\text { Annual }\end{array}$ & Field & Low & $\begin{array}{l}\text { DEM, a land } \\
\text { use map, and } \\
\text { the major tillage } \\
\text { direction on } \\
\text { each field }\end{array}$ & $\begin{array}{l}\text { Incorporated } \\
\text { with LISEM } \\
\text { model, } \\
\text { Generalized } \\
\text { erosion- } \\
\text { deposition } \\
\text { mass balance, } \\
\text { Dynamic } \\
\text { Erosion } \\
\text { concept } \\
\text { eqn. } \\
\end{array}$ & $\begin{array}{l}\text { Runoff } \\
\text { pattern, } \\
\text { erosion } \\
\text { patterns, } \\
\text { runoff } \\
\text { network }\end{array}$ & $\begin{array}{c}\text { Local } \\
\text { depressions that } \\
\text { may exist in a } \\
\text { DEM need to be } \\
\text { removed } \\
\text { making runoff } \\
\text { pattern more } \\
\text { complicated }\end{array}$ & No & No & No & No & [33] \\
\hline 8 & TMDL & $\begin{array}{l}\text { Total } \\
\text { Maximum } \\
\text { Daily Load }\end{array}$ & $\begin{array}{c}\text { USA EPA } \\
\text { (1991) }\end{array}$ & Annual & Catchment & High & $\begin{array}{l}\text { Channel } \\
\text { network, } \\
\text { Groundwater } \\
\text { exchange, } \\
\text { Topography, } \\
\text { unit discharge } \\
\text { rate, soil } \\
\text { cropping factor, } \\
\text { conservation } \\
\text { factor }\end{array}$ & $\begin{array}{l}\text { Modified } \\
\text { Kilinc- } \\
\text { Richardson } \\
\text { equation for } \\
\text { soil erosion, } \\
\text { advection- } \\
\text { dispersion } \\
\text { equation for } \\
\text { in-channel } \\
\text { sediment } \\
\text { transporta- } \\
\text { tion, general } \\
\text { transport } \\
\text { equation for } \\
\text { overland } \\
\text { sediment } \\
\text { transport }\end{array}$ & $\begin{array}{l}\text { Multi- } \\
\text { dimensional, } \\
\text { Provides } \\
\text { amount of } \\
\text { sediment and } \\
\text { nutrients }\end{array}$ & $\begin{array}{l}\text { Transport } \\
\text { capacity must } \\
\text { be converted } \\
\text { into erosion } \\
\text { coefficient. } \\
\text { determining the } \\
\text { interdependent } \\
\text { factors is } \\
\text { difficult. }\end{array}$ & No & No & No & No & [34] \\
\hline
\end{tabular}


Table 3. Cont.

\begin{tabular}{|c|c|c|c|c|c|c|c|c|c|c|c|c|c|c|c|}
\hline \multirow{2}{*}{ Sr. No. } & \multirow{2}{*}{ Model } & \multirow{2}{*}{ Description } & \multirow{2}{*}{$\begin{array}{c}\text { Developer } \\
\text { /Year * }\end{array}$} & \multicolumn{2}{|c|}{ Scale } & \multicolumn{2}{|c|}{ Input } & \multirow{2}{*}{$\begin{array}{l}\text { Governing } \\
\text { Equations }\end{array}$} & \multirow{2}{*}{$\begin{array}{l}\text { Model } \\
\text { Capabilities }\end{array}$} & \multirow{2}{*}{$\begin{array}{c}\text { Model } \\
\text { Limitations }\end{array}$} & \multicolumn{3}{|c|}{$\begin{array}{c}\text { Overland } \\
\text { Sedimentation }\end{array}$} & \multirow{2}{*}{$\begin{array}{c}\text { Channel } \\
\text { Sedimentation } \\
\text { Generation }\end{array}$} & \multirow{2}{*}{ Source } \\
\hline & & & & Temporal & Spatial & Demand & Variables & & & & $\mathrm{G}^{*}$ & $T^{*}$ & $\mathbf{D}^{*}$ & & \\
\hline 9 & SLEMSA & $\begin{array}{l}\text { Soil Loss } \\
\text { Estimation } \\
\text { Model } \\
\text { for Southern } \\
\text { Africa }\end{array}$ & [35] & Annual & Field & High & $\begin{array}{l}\text { Climate data, } \\
\text { topography, } \\
\text { vegetation, } \\
\text { human } \\
\text { influence }\end{array}$ & $\begin{array}{l}\text { ELWELL } \\
\text { equation } \\
Z=K^{*} X^{*} C \\
\text { Where } \mathrm{K} \\
\text { (Mean annual } \\
\text { soil loss } \\
\text { index, } \\
X \\
\text { (Topographic } \\
\text { Factor), } C \\
\text { (Crop cover/ } \\
\text { management } \\
\text { factor) }\end{array}$ & $\begin{array}{l}\text { Soil erosion, } \\
\text { decision on } \\
\text { land } \\
\text { management } \\
\text { techniques }\end{array}$ & $\begin{array}{l}\text { High } \\
\text { sensitivity to } \\
\text { the input } \\
\text { factors }\end{array}$ & No & Yes & No & No & [24] \\
\hline 10 & PSIAC & $\begin{array}{l}\text { Pacific } \\
\text { Southwest } \\
\text { Inter-agency } \\
\text { Committee } \\
\text { Method }\end{array}$ & [36] & Annual & $\begin{array}{l}\text { Catchment/ } \\
\text { Field }\end{array}$ & High & $\begin{array}{l}\text { Surface geology, } \\
\text { soil types, } \\
\text { Climate, slope, } \\
\text { stream, } \\
\text { network, land } \\
\text { cover/land use }\end{array}$ & $\begin{array}{l}\text { Gravelius } \\
\text { Equation, } \\
\text { Horton } \\
\text { Equation, } \\
\text { Kiripich } \\
\text { Equation, } \\
\text { Drainage } \\
\text { Density } \\
\text { Equation, } \\
\text { upland } \\
\text { erosion = } \\
0.25 S S F \text { (SSF: } \\
\text { soil surface } \\
\text { factor), } \\
\text { Channel } \\
\text { erosion = } \\
\text { 1.67SSFg } \\
\text { (SSFg: Gully } \\
\text { erosion } \\
\text { factor) }\end{array}$ & $\begin{array}{l}\text { Upland } \\
\text { erosion, } \\
\text { Channel } \\
\text { erosion, } \\
\text { sediment } \\
\text { deposition }\end{array}$ & $\begin{array}{c}\text { Model } \\
\text { sensitivity to } \\
\text { changes of } \\
\text { different factors } \\
\text { under different } \\
\text { conditions }\end{array}$ & Yes & Yes & Yes & Yes & [37] \\
\hline 11 & $\mathrm{E}_{30}$ & $\begin{array}{l}\text { Soil Erosion } \\
\text { at } 30^{\circ} \text { slope }\end{array}$ & [38] & Annual & Watershed & Low & $\begin{array}{l}\text { Land use/Land } \\
\text { cover maps, } \\
\text { topography }\end{array}$ & $\begin{array}{l}E=E_{30}{ }^{*} \\
\left(\mathrm{~S} / \mathrm{S}_{30}\right)^{0.9} \\
\mathrm{E} \text { : rate of soil } \\
\text { erosion; } \\
\text { E30: rate of } \\
\text { soil } \\
\text { erosion at } 30^{0} \\
\text { slope; S30: } \\
\text { 300 slope }\end{array}$ & Soil Erosion & $\begin{array}{c}\text { Model applies } \\
\text { only to hilly } \\
\text { regions having } \\
\text { undulant } \\
\text { topography and } \\
\text { steep slopes. } \\
\text { Does not take } \\
\text { into account soil } \\
\text { factors (crirtical } \\
\text { for erosion } \\
\text { processes) }\end{array}$ & No & Yes & No & No & [38] \\
\hline
\end{tabular}


Table 3. Cont.

\begin{tabular}{|c|c|c|c|c|c|c|c|c|c|c|c|c|c|c|c|}
\hline \multirow{2}{*}{ Sr. No. } & \multirow{2}{*}{ Model } & \multirow{2}{*}{ Description } & \multirow{2}{*}{$\begin{array}{c}\text { Developer } \\
\text { /Year }{ }^{*}\end{array}$} & \multicolumn{2}{|c|}{ Scale } & \multicolumn{2}{|c|}{ Input } & \multirow{2}{*}{$\begin{array}{l}\text { Governing } \\
\text { Equations }\end{array}$} & \multirow{2}{*}{$\begin{array}{l}\text { Model } \\
\text { Capabilities }\end{array}$} & \multirow{2}{*}{$\begin{array}{c}\text { Model } \\
\text { Limitations }\end{array}$} & \multicolumn{3}{|c|}{$\begin{array}{c}\text { Overland } \\
\text { Sedimentation }\end{array}$} & \multirow{2}{*}{$\begin{array}{c}\text { Channel } \\
\text { Sedimentation } \\
\text { Generation }\end{array}$} & \multirow{2}{*}{ Sourc } \\
\hline & & & & Temporal & Spatial & Demand & Variables & & & & $\mathrm{G}^{*}$ & $\mathrm{~T}^{*}$ & $\mathrm{D}^{*}$ & & \\
\hline 12 & WaNuLCAS & $\begin{array}{l}\text { water, } \\
\text { nutrient and } \\
\text { light capture } \\
\text { in } \\
\text { agroforestry } \\
\text { system }\end{array}$ & [39] & Annual & $\begin{array}{l}\text { Watershed } \\
\text { /field }\end{array}$ & High & $\begin{array}{l}\text { Land use/Land } \\
\text { cover, Climate } \\
\text { data }\end{array}$ & USLE & $\begin{array}{l}\text { Soil erosion, } \\
\text { crop yield }\end{array}$ & $\begin{array}{l}\text { The erosion } \\
\text { component is } \\
\text { not well } \\
\text { developed and } \\
\text { integrated with } \\
\text { crop yield }\end{array}$ & Yes & No & No & No & [40] \\
\hline 13 & SCUAF & $\begin{array}{l}\text { Soil Changes } \\
\text { Under } \\
\text { Agroforestry }\end{array}$ & [41] & $\begin{array}{l}\text { Annual// } \\
\text { Seasonal }\end{array}$ & $\begin{array}{l}\text { Watershed } \\
\text { /field }\end{array}$ & High & $\begin{array}{l}\text { Crop, soil } \\
\text { physical and } \\
\text { chemical } \\
\text { properties }\end{array}$ & USLE & $\begin{array}{l}\text { Predict soil } \\
\text { changes } \\
\text { under } \\
\text { different } \\
\text { agroforestry } \\
\text { systems }\end{array}$ & $\begin{array}{l}\text { Erosion } \\
\text { component } \\
\text { is not well } \\
\text { tested }\end{array}$ & No & No & No & No & [41] \\
\hline
\end{tabular}

$\mathrm{G}^{*}$ : Generation; $\mathrm{T}$ *: Transportation; $\mathrm{D}$ *: Deposition; Developer/year *: references for model manuals and first research articles describing the respective model. 


\subsubsection{Conceptual Models}

Conceptual models are based on the sediment and runoff continuity equations, and basically take a position between physically based and empirical models [42]. Unlike empirical models, conceptual models reflect the process governing the system behavior. The primary focus of conceptual models has been to estimate sediment yield based on the concept of unit hydrograph [43]. Therefore, they typically consider the most critical catchment characteristics and corresponding soil erosion processes, however, without describing the details of these processes and interactions that would require data on temporal and spatially distributed catchment details [44]. As a result, conceptual models can be used to simulate quantitative and qualitative impacts of land use changes on soil erosion and sediment yields without having to be parametrized with detailed catchment information.

Jakeman et al. [45] noted that conceptual models tend to have issues related to the identifiability of their parameter values since those values were generally obtained during model calibration with observed values [46]. Sorooshian et al. 1991 [44] identified the direct relationship between conceptual model complexity and model identification. The calibration procedure for medium complex models can find only the local best fit although there may be many other local conditions with optimum parameter sets. This problem can be resolved by reducing the number of parameters that have to be estimated through calibration and increasing the number of parameters that can be estimated based on prior knowledge of the system [47]. Such an approach will reduce the goodness of fit to the calibration data. The lack of parameter values for conceptual models means limiting the physical interpretability of parameters [48]. Though more complex models tend to offer a better fit to calibration data they also carry the risk of over-fitting when calibration data are limited [49].

Most of the conceptual models use equations from empirical approaches (Table 5). The empirical models USLE and MUSLE, for example, are implemented in conceptual models such as APSIM (modeling framework), SWIM, RillGrow, SWRRB, LASCAM for estimating soil erosion. These conceptual models can predict the temporal and spatial distribution of soil detachment and sedimentation at a field scale depending on crop and soil management at daily time steps. Among these models, APSIM and IQQM are continuous simulation models predicting both overland and channel sediment generation, transportation and deposition, as well as rainfall-runoff associated nutrient loss and soil changes. A few models such as APSIM, AGNPS, AGNPS-UM are event-based models to predict soil erosion under complex agriculture systems from smaller scales (hill-slopes) to large (catchment) scales (Table 5). 
Table 4. Conceptual soil erosion models

\begin{tabular}{|c|c|c|c|c|c|c|c|c|c|c|c|c|c|c|c|}
\hline \multirow{2}{*}{ Sr. No. } & \multirow{2}{*}{ Model } & \multirow{2}{*}{ Description } & \multirow{2}{*}{$\begin{array}{c}\text { Developer } \\
\text { /Year* }\end{array}$} & \multicolumn{2}{|c|}{ Scale } & \multicolumn{2}{|c|}{ Input } & \multirow{2}{*}{$\begin{array}{l}\text { Governing } \\
\text { Equations }\end{array}$} & \multirow{2}{*}{$\begin{array}{l}\text { Model } \\
\text { Capabilities }\end{array}$} & \multirow{2}{*}{$\begin{array}{l}\text { Model } \\
\text { Limitations }\end{array}$} & \multicolumn{3}{|c|}{$\begin{array}{c}\text { Overland } \\
\text { Sedimentation }\end{array}$} & \multirow{2}{*}{$\begin{array}{c}\text { Channel } \\
\text { Sedimentation } \\
\text { Generation }\end{array}$} & \multirow{2}{*}{ Source } \\
\hline & & & & Temporal & Spatial & Demand & Variables & & & & $\mathrm{G}^{*}$ & $\mathrm{~T}^{*}$ & $\mathrm{D}^{*}$ & & \\
\hline 1 & APSIM & $\begin{array}{l}\text { Agricultural } \\
\text { Production } \\
\text { Simulator }\end{array}$ & [50] & Daily & Field & High & $\begin{array}{l}\text { Climate, } \\
\text { topography, } \\
\text { land use, crop, } \\
\text { field } \\
\text { management } \\
\text { practices }\end{array}$ & $\begin{array}{l}\text { Modified } \\
\text { USLE, soil } \\
\text { water balance } \\
\text { equation }\end{array}$ & Erosion, ${ }^{*}$ & $\begin{array}{l}\text { Intensive } \\
\text { calibration and } \\
\text { validation } \\
\text { required }\end{array}$ & Yes & Yes & Yes & No & [51] \\
\hline 2 & RillGrow & RillGrow & [52] & Abstract & Plot & High & $\begin{array}{l}\text { Meteorology, } \\
\text { Digital Terrain } \\
\text { Model }\end{array}$ & $\begin{array}{l}\text { S-Curve } \\
\text { stream } \\
\text { power based } \\
\text { equations }\end{array}$ & $\begin{array}{l}\text { Formation } \\
\text { and } \\
\text { simulation of } \\
\text { rill network }\end{array}$ & $\begin{array}{l}\text { Depends on } \\
\text { single storm } \\
\text { events; Low } \\
\text { potential for } \\
\text { integration with } \\
\text { GIS }\end{array}$ & Yes & Yes & Yes & No & [52] \\
\hline 3 & SWAT & $\begin{array}{l}\text { Soil and } \\
\text { Water } \\
\text { Assessment } \\
\text { Tool }\end{array}$ & [53] & Daily & $\begin{array}{l}\text { Regional to } \\
\text { watershed }\end{array}$ & Medium & $\begin{array}{l}\text { Climate, soil } \\
\text { characteristics, } \\
\text { topography, } \\
\text { land use / Land } \\
\text { cover }\end{array}$ & $\begin{array}{l}\text { MUSLE, } \\
\text { Manning's } \\
\text { equation, SCS } \\
\text { Curve } \\
\text { Number, } \\
\text { Bagnold's } \\
\text { stream power } \\
\text { Concept, } \\
\text { Continuity } \\
\text { equation }\end{array}$ & $\begin{array}{l}\text { Hydrological } \\
\text { assessments, } \\
\text { pollutant loss } \\
\text { studies, } \\
\text { water erosion, } \\
\text { sediment } \\
\text { yield }\end{array}$ & $\begin{array}{l}\text { Weak in stream } \\
\text { channel } \\
\text { degradation } \\
\text { and sediment } \\
\text { deposition } \\
\text { analysis, } \\
\text { inadequate data } \\
\text { availability for } \\
\text { calibration and } \\
\text { validation }\end{array}$ & Yes & Yes & Yes & Yes & [54] \\
\hline 4 & SWIM & $\begin{array}{l}\text { Soil and } \\
\text { Water } \\
\text { Integrated } \\
\text { Model }\end{array}$ & [55] & Daily & Watershed & Medium & $\begin{array}{l}\text { Climate, soil } \\
\text { characteristics, } \\
\text { land } \\
\text { cover, crop } \\
\text { types }\end{array}$ & $\begin{array}{l}\text { water balance } \\
\text { equation, } \\
\text { MUSLE, SCS } \\
\text { Curve } \\
\text { Number, }\end{array}$ & $\begin{array}{l}\text { Simulation of } \\
\text { runoff, } \\
\text { soil erosion, } \\
\text { sedimenta- } \\
\text { tion } \\
*\end{array}$ & $\begin{array}{l}\text { relatively } \\
\text { complex, no } \\
\text { simulation of } \\
\text { gully erosion }\end{array}$ & Yes & No & No & No & [56] \\
\hline 5 & IQQM & $\begin{array}{l}\text { Integrated } \\
\text { Water } \\
\text { Quality and } \\
\text { Quantity } \\
\text { Model }\end{array}$ & [57] & Daily & Watershed & Medium & $\begin{array}{l}\text { Topography, } \\
\text { river system } \\
\text { configuration, } \\
\text { evapotranspira- } \\
\text { tion }\end{array}$ & $\begin{array}{l}\text { conceptual } \\
\text { Sacramento } \\
\text { model, } \\
\text { QUAL2E } \\
\text { model }\end{array}$ & $\begin{array}{l}\text { Rainfall- } \\
\text { runoff } \\
\text { generation, * }\end{array}$ & $\begin{array}{l}\text { No erosion or } \\
\text { sediment } \\
\text { generation } \\
\text { simulation }\end{array}$ & Yes & No & No & Yes & [57] \\
\hline 6 & CAESAR & $\begin{array}{l}\text { Cellular } \\
\text { Automaton } \\
\text { Evolutionary } \\
\text { Slope and } \\
\text { River } \\
\text { model }\end{array}$ & [58] & Annual & Catchment & High & $\begin{array}{l}\text { DEM, Rainfall, } \\
\text { flow } \\
\text { parameters, } \\
\text { slope processes, } \\
\text { bedrock depth, } \\
\text { value of } \\
\text { Manning } \\
\text { coefficient }\end{array}$ & $\begin{array}{l}\text { Einstein } \\
\text { equation, } \\
\text { Wilcock } \\
\text { \& Crowe } \\
\text { equations }\end{array}$ & $\begin{array}{l}\text { Erosion, } \\
\text { sediment } \\
\text { transport \& } \\
\text { deposition }\end{array}$ & $\begin{array}{l}\text { No } \\
\text { rainfall-runoff } \\
\text { interactoin }\end{array}$ & Yes & Yes & Yes & Yes & [59] \\
\hline
\end{tabular}


Table 4. Cont

\begin{tabular}{|c|c|c|c|c|c|c|c|c|c|c|c|c|c|c|c|}
\hline \multirow{2}{*}{ Sr. No. } & \multirow{2}{*}{ Model } & \multirow{2}{*}{ Description } & \multirow{2}{*}{$\begin{array}{l}\text { Developer } \\
\text { /Year* }\end{array}$} & \multicolumn{2}{|c|}{ Scale } & \multicolumn{2}{|c|}{ Input } & \multirow{2}{*}{$\begin{array}{l}\text { Governing } \\
\text { Equations }\end{array}$} & \multirow{2}{*}{$\begin{array}{l}\text { Model } \\
\text { Capabilities }\end{array}$} & \multirow{2}{*}{$\begin{array}{l}\text { Model } \\
\text { Limitations }\end{array}$} & \multicolumn{3}{|c|}{$\begin{array}{c}\text { Overland } \\
\text { Sedimentation }\end{array}$} & \multirow{2}{*}{$\begin{array}{c}\text { Channel } \\
\text { Sedimentation } \\
\text { Generation }\end{array}$} & \multirow{2}{*}{ Source } \\
\hline & & & & Temporal & Spatial & Demand & Variables & & & & $\mathrm{G}^{*}$ & $T^{*}$ & $\mathbf{D}^{*}$ & & \\
\hline 7 & TOPMODEL & $\begin{array}{l}\text { Topography } \\
\text { based } \\
\text { hydrological } \\
\text { MODEL) }\end{array}$ & {$[60,61]$} & Daily & Hillslope & Medium & $\begin{array}{l}\text { DEM, landform } \\
\text { features, soil } \\
\text { characteristics, } \\
\text { geology, } \\
\text { vegetation, and } \\
\text { hydrological } \\
\text { characteristics }\end{array}$ & $\begin{array}{l}\text { Sediment } \\
\text { transport } \\
\text { capacity, } \\
\text { continuity } \\
\text { equation }\end{array}$ & $\begin{array}{l}\text { Soil moisture } \\
\text { deficit, } \\
\text { rainfall- } \\
\text { runoff, } \\
\text { Simulation of } \\
\text { surface/ } \\
\text { subsurface } \\
\text { hydrology; } \\
\text { sediment } \\
\text { yield } \\
\text { and transport }\end{array}$ & $\begin{array}{l}\text { Suitable only } \\
\text { for shallow } \\
\text { homogenous } \\
\text { soil watersheds }\end{array}$ & Yes & No & No & No & [62] \\
\hline 8 & WILSIM & $\begin{array}{l}\text { Web-based } \\
\text { Interactive } \\
\text { Landform } \\
\text { Simulation } \\
\text { Model }\end{array}$ & [63] & Abstract & Watershed & High & $\begin{array}{l}\text { DEM, } \\
\text { Topography, } \\
\text { Rainfall, flow } \\
\text { parameters, } \\
\text { slope }\end{array}$ & $\begin{array}{l}\text { Cellular } \\
\text { automata } \\
\text { (CA) } \\
\text { algorithm }\end{array}$ & $\begin{array}{l}\text { simulation } \\
\text { offers an ideal } \\
\text { tool for un- } \\
\text { derstanding } \\
\text { the complex } \\
\text { effects of a } \\
\text { variety of } \\
\text { physical and } \\
\text { geological } \\
\text { processes and } \\
\text { erosion }\end{array}$ & $\begin{array}{l}\text { Many details of } \\
\text { the physical } \\
\text { process are not } \\
\text { included in the } \\
\text { model. }\end{array}$ & Yes & No & No & Yes & [64] \\
\hline 10 & LASCAM & $\begin{array}{l}\text { Large Scale } \\
\text { Catchment } \\
\text { Model }\end{array}$ & [66] & Daily & Catchment & High & $\begin{array}{l}\text { Sediment load, } \\
\text { runoff, salt } \\
\text { fluxes }\end{array}$ & $\begin{array}{l}\text { USLE, Stream } \\
\text { sediment } \\
\text { capacity }\end{array}$ & $\begin{array}{l}\text { Simulation of } \\
\text { hydrology, } \\
\text { erosion, }\end{array}$ & $\begin{array}{l}\text { During } \\
\text { calibration low } \\
\text { quality of } \\
\text { sediment and } \\
\text { nutrient } \\
\text { predictions }\end{array}$ & Yes & Yes & Yes & Yes & [67] \\
\hline 11 & AGNPS & $\begin{array}{l}\text { Agricultural } \\
\text { Non-Point } \\
\text { Source } \\
\text { pollution } \\
\text { model }\end{array}$ & [68] & Daily & $\begin{array}{l}\text { Small- to } \\
\text { medium- } \\
\text { sized } \\
\text { watersheds }\end{array}$ & High & $\begin{array}{l}\text { Climate, } \\
\text { topography, soil } \\
\text { characteristics, } \\
\text { Land use }\end{array}$ & $\begin{array}{l}\text { SCS Curve } \\
\text { Number, } \\
\text { USLE, } \\
\text { Foster } \\
\text { equation }\end{array}$ & $\begin{array}{l}\text { Soil erosion, } \\
\text { sediment } \\
\text { transport and } \\
\text { depositing, }\end{array}$ & $\begin{array}{l}\text { Does not } \\
\text { simulate } \\
\text { sub-surface } \\
\text { flow, only } \\
\text { suitable to } \\
\text { small-medium } \\
\text { catchments }\end{array}$ & Yes & No & No & Yes & [37] \\
\hline
\end{tabular}


Table 4. Cont.

\begin{tabular}{|c|c|c|c|c|c|c|c|c|c|c|c|c|c|c|c|}
\hline \multirow{2}{*}{ Sr. No. } & \multirow{2}{*}{ Model } & \multirow[t]{2}{*}{ Description } & \multirow{2}{*}{$\begin{array}{c}\text { Developer } \\
\text { /Year }{ }^{*}\end{array}$} & \multicolumn{2}{|c|}{ Scale } & \multicolumn{2}{|c|}{ Input } & \multirow{2}{*}{$\begin{array}{l}\text { Governing } \\
\text { Equations }\end{array}$} & \multirow{2}{*}{$\begin{array}{l}\text { Model } \\
\text { Capabilities }\end{array}$} & \multirow{2}{*}{$\begin{array}{l}\text { Model } \\
\text { Limitations }\end{array}$} & \multicolumn{3}{|c|}{$\begin{array}{c}\text { Overland } \\
\text { Sedimentation }\end{array}$} & \multirow{2}{*}{$\begin{array}{c}\text { Channel } \\
\text { Sedimentation } \\
\text { Generation }\end{array}$} & \multirow{2}{*}{ Source } \\
\hline & & & & Temporal & Spatial & Demand & Variables & & & & $\mathrm{G}^{*}$ & $\mathrm{~T}^{*}$ & $\mathrm{D}^{*}$ & & \\
\hline 12 & ACRU & $\begin{array}{l}\text { Agricultural } \\
\text { Catchment } \\
\text { Research Unit }\end{array}$ & [69] & Daily & $\begin{array}{c}\text { Small } \\
\text { catchments } \\
\left(<10 \mathrm{~km}^{2}\right)\end{array}$ & Low & $\begin{array}{l}\text { Climate, soil, } \\
\text { land use crop }\end{array}$ & $\begin{array}{l}\text { SCS equation, } \\
\text { catchment } \\
\text { curve } \\
\text { number, }\end{array}$ & $\begin{array}{l}\text { Simulate } \\
\text { runoff, } \\
\text { erosion and } \\
\text { sediment } \\
\text { yield, land } \\
\text { use and } \\
\text { climate } \\
\text { impacts, } \\
\text { seasonal crop } \\
\text { yield }\end{array}$ & $\begin{array}{l}\text { Require } \\
\text { extensive GIS } \\
\text { pre-processing }\end{array}$ & Yes & No & No & Yes & [70] \\
\hline 13 & STREAM & $\begin{array}{l}\text { Sealing, } \\
\text { Transfer, } \\
\text { Runoff, } \\
\text { Erosion, } \\
\text { Agricultural } \\
\text { Modification } \\
\text { model }\end{array}$ & [71] & Event & $\begin{array}{c}\text { Catchment } \\
\text { to } \\
\text { watershed }\end{array}$ & High & $\begin{array}{l}\text { rainfall, } \\
\text { temperature, } \\
\text { topography, soil } \\
\text { (water holding } \\
\text { capacity), land } \\
\text { cover }\end{array}$ & USLE & $\begin{array}{l}\text { Simulates } \\
\text { land use } \\
\text { impacts, } \\
\text { erosion, sedi- } \\
\text { mentation }\end{array}$ & $\begin{array}{l}\text { applicable to } \\
\text { single rainfall } \\
\text { events }\end{array}$ & Yes & Yes & Yes & Yes & [72] \\
\hline 14 & $\begin{array}{l}\text { AGNPS- } \\
\text { UM }\end{array}$ & $\begin{array}{l}\text { Agricultural } \\
\text { Non-Point } \\
\text { Source } \\
\text { pollution } \\
\text { model, }\end{array}$ & [73] & Daily & $\begin{array}{c}\text { Catchment } \\
\text { to } \\
\text { watershed }\end{array}$ & High & $\begin{array}{l}\text { Climate, } \\
\text { topography, soil } \\
\text { characteristics, } \\
\text { Land use }\end{array}$ & USLE-M & $\begin{array}{l}\text { Management } \\
\text { decisions on } \\
\text { water and } \\
\text { sediment } \\
\text { yields }\end{array}$ & $\begin{array}{l}\text { Rely on } \\
\text { single storm } \\
\text { event; data } \\
\text { intensive }\end{array}$ & Yes & No & No & Yes & [73] \\
\hline
\end{tabular}

* additional model capabilities besides soil erosion; G: Generation; T: Transportation; D: Deposition; Developer/year: references for model manuals and first research articles describing respective model. 


\subsubsection{Physically based Models}

In general, physically based soil erosion models are based on the fundamental concepts of physics using conservation of momentum, energy, and mass as governing equations $[74,75]$ that are solved by various numerical techniques. Thus, these models consist of multiple equations and algorithms and a large number of parameters to simulate and predict the dynamics of soil erosion and sedimentation rates. They explicitly simulate the water fluxes, e.g., overland flow based on the kinematic wave theory [76], and apply the kinematic wave theory based on continuity and momentum equations. The continuity equation refers to the balance between inflow into the system and change in system storage and the momentum equation represents the pressure gradient between energy gradient and surface slope. Other most famous approaches for simulating the water fluxes include the Manning's and Chézy's equations in large watersheds (Table 5).

In general, the equations of individual model components in physically based models are based on a large number of assumptions that may not be relevant in the real world [77]. These governing equations were often developed under controlled conditions using continuous data observed at single observation points or small spatial scales [78]. In practice, these equations are applied for grid cells representing much larger areas of watersheds with varying physical conditions. Corresponding assumptions required for upscaling point-based observations may compromise the physical significance of models [79]. Merritt et al. [5] pointed out that there is not enough evidence on the suitability of these equations for modeling water erosion beyond a small field scale. Pechlivanidis et al. [48], therefore, suggested applying simplified computation techniques to represent individual processes which avoid unwanted deflection from real field scenarios and additional uncertainties. In practice, parameters used in physically based models should be calibrated with observed data that, on the other hand, creates a lack of identifiability analysis of optimum parameters and distinctiveness of best fit to the veracity of modeling outputs [80]. Model comparisons illustrate that the application of physically based models (e.g., AGNPS or PESERA) does not necessarily result in lower uncertainties compared to more simple structured empirical models such as USLE-type algorithms.

Physically based field-scale models such as EPIC, EGEM, CREAMS, EROSION 2D/3D, GUEST, GLEAMS, MEFDIS, MEDALUS, PERFECT, PEPP-HILLFLOW, etc., are more capable of responding to event-based or continuous storms to simulate surface runoff, soil detachment, transportation, and sediment yield (Table 5). The EPIC model considers the effect of several best management practices (BMPs) related to crop, soil, and nutrient management on soil erosion and soil productivity. CREAMS is another model that is used for describing the hydrology, erosion, and sediment size distribution as well as changes in soil depth, chemical, nutrient, and sediment yield for field-scale croplands. Productivity, Erosion and Runoff, Functions to Evaluate Conservation Techniques (PERFECT) is a dynamic model suitable for event-based analysis of soil erosion and surface runoff over a small scale. This model can also be integrated with a GIS tool for visualization of results. The main disadvantage of this model is that it overestimates the outputs of surface runoff and surface water retention capacity as influenced by complex tillage patterns and tillage directions. 
Table 5. Physically based soil erosion models.

\begin{tabular}{|c|c|c|c|c|c|c|c|c|c|c|c|c|c|c|c|}
\hline \multirow{2}{*}{ Sr. No. } & \multirow{2}{*}{ Model } & \multirow{2}{*}{ Description } & \multirow{2}{*}{$\begin{array}{c}\text { Developer } \\
\text { /Year* }\end{array}$} & \multicolumn{2}{|c|}{ Scale } & \multicolumn{2}{|c|}{ Input } & \multirow{2}{*}{$\begin{array}{l}\text { Governing } \\
\text { Equations }\end{array}$} & \multirow{2}{*}{$\begin{array}{l}\text { Model } \\
\text { Capabilities }\end{array}$} & \multirow{2}{*}{$\begin{array}{l}\text { Model } \\
\text { Limitations }\end{array}$} & \multicolumn{3}{|c|}{$\begin{array}{c}\text { Overland } \\
\text { Sedimentation }\end{array}$} & \multirow{2}{*}{$\begin{array}{c}\text { Channel } \\
\text { Sedimentation } \\
\text { Generation }\end{array}$} & \multirow{2}{*}{ Source } \\
\hline & & & & Temporal & Spatial & Demand & Variables & & & & $\mathrm{G}^{*}$ & $\mathrm{~T}^{*}$ & $\mathrm{D}^{*}$ & & \\
\hline 1 & ANSWERS & $\begin{array}{l}\text { Areal } \\
\text { Nonpoint } \\
\text { Source } \\
\text { Watershed } \\
\text { Environment } \\
\text { Response } \\
\text { Simulation }\end{array}$ & [81] & Event & $\begin{array}{l}\text { Regional to } \\
\text { small } \\
\text { catchment }\end{array}$ & High & $\begin{array}{l}\text { Climate, soil } \\
\text { characteristics, } \\
\text { topography, } \\
\text { land } \\
\text { use, drainage } \\
\text { network, field } \\
\text { management } \\
\text { practices }\end{array}$ & $\begin{array}{l}\text { USLE, } \\
\text { steady-state } \\
\text { sediment } \\
\text { continuity } \\
\text { equation, } \\
\text { Modified } \\
\text { Yalin } \\
\text { equation, } \\
\text { Foster } \\
\text { equation }\end{array}$ & $\begin{array}{l}\text { Erosion, } \\
\text { sediment } \\
\text { yield, runoff, } \\
\text { peak flow } \\
\text { rate, } \\
\text { nutrients, }{ }^{*}\end{array}$ & $\begin{array}{l}\text { Relies on single } \\
\text { storm } \\
\text { Event, consider } \\
\text { erodibility as } \\
\text { time constant } \\
\text { parameter }\end{array}$ & Yes & Yes & Yes & No & [82] \\
\hline 2 & EPIC & $\begin{array}{l}\text { Erosion- } \\
\text { Productivity } \\
\text { Impact } \\
\text { Calculator }\end{array}$ & [83] & Daily & $\begin{array}{l}\text { Plots to } \\
\text { field-sized } \\
\text { areas }\end{array}$ & High & $\begin{array}{l}\text { Hydrology, } \\
\text { meteorology, } \\
\text { erosion, } \\
\text { nutrients, plant } \\
\text { growth, soil } \\
\text { temperature, } \\
\text { and tillage. }\end{array}$ & $\begin{array}{l}\text { curve number } \\
\text { equation, } \\
\text { Onstad- } \\
\text { Foster } \\
\text { equation, } \\
\text { USLE, } \\
\text { MULSE }\end{array}$ & $\begin{array}{l}\text { Surface } \\
\text { runoff, } \\
\text { sediment } \\
\text { yield, soil } \\
\text { erosion* }\end{array}$ & $\begin{array}{l}\text { Applicable to } \\
\text { only field scale, } \\
\text { less } \\
\text { incorporation } \\
\text { with GIS tools }\end{array}$ & Yes & Yes & Yes & Yes & [84] \\
\hline 3 & $\begin{array}{l}\text { ANSWERS- } \\
\text { continuous }\end{array}$ & $\begin{array}{l}\text { Areal } \\
\text { Nonpoint } \\
\text { Source } \\
\text { watershed } \\
\text { Environment } \\
\text { Response } \\
\text { Simulation- } \\
\text { Continuous } \\
\end{array}$ & [82] & Event & $\begin{array}{l}\text { Regional to } \\
\text { small } \\
\text { catchment }\end{array}$ & High & $\begin{array}{l}\text { Climate, soil } \\
\text { characteristics, } \\
\text { topography, } \\
\text { land } \\
\text { use, drainage } \\
\text { network, field } \\
\text { management } \\
\text { practices }\end{array}$ & $\begin{array}{l}\text { USLE, } \\
\text { Modified } \\
\text { Yalin } \\
\text { equation, } \\
\text { Foster } \\
\text { equation, } \\
\text { Manning's } \\
\text { equation }\end{array}$ & $\begin{array}{l}\text { Erosion, } \\
\text { sediment } \\
\text { yield, }^{*}\end{array}$ & $\begin{array}{l}\text { No simulation } \\
\text { of channel } \\
\text { sediment }\end{array}$ & Yes & Yes & Yes & No & [82] \\
\hline 4 & EGEM & $\begin{array}{l}\text { (Ephemeral } \\
\text { Gully Erosion } \\
\text { Model }\end{array}$ & [85] & Event & $\begin{array}{l}\text { Field to } \\
\text { small } \\
\text { catchment }\end{array}$ & Medium & $\begin{array}{l}\text { Rainfall, soil } \\
\text { characteristics, } \\
\text { Topography }\end{array}$ & $\begin{array}{l}\text { Physical- } \\
\text { process } \\
\text { equations } \\
\text { CREAMS } \\
\text { empirical } \\
\text { relationship }\end{array}$ & $\begin{array}{l}\text { Annual } \\
\text { estimation of } \\
\text { Transient } \\
\text { gully erosion }\end{array}$ & $\begin{array}{l}\text { Requires } \\
\text { intensive } \\
\text { watershed } \\
\text { information }\end{array}$ & Yes & No & No & Yes & [86] \\
\hline 5 & DWSM & $\begin{array}{l}\text { Dynamic } \\
\text { Watershed } \\
\text { Simulation } \\
\text { Model }\end{array}$ & [87] & Event & Catchment & High & $\begin{array}{l}\text { Stream network, } \\
\text { watershed } \\
\text { hydrology, } \\
\text { water quality, } \\
\text { land use }\end{array}$ & $\begin{array}{l}\text { continuity } \\
\text { equation }\end{array}$ & $\begin{array}{l}\text { Simulation of } \\
\text { erosion, } \\
\text { runoff, } \\
\text { erosion, } \\
\text { sediment } \\
\text { yield * }\end{array}$ & $\begin{array}{l}\text { Slow } \\
\text { computing } \\
\text { speed, } \\
\text { uncertainties in } \\
\text { input parameter } \\
\text { data }\end{array}$ & Yes & Yes & Yes & Yes & [87] \\
\hline 6 & CREAMS & $\begin{array}{l}\text { Chemicals, } \\
\text { Runoff and } \\
\text { Erosion from } \\
\text { Agricultural } \\
\text { Management } \\
\text { Systems }\end{array}$ & [88] & Monthly & $\begin{array}{l}\text { Plot to } \\
\text { Field }\end{array}$ & High & $\begin{array}{l}\text { Climate, } \\
\text { vegetation, } \\
\text { cultural } \\
\text { practices }\end{array}$ & $\begin{array}{l}\text { Foster } \\
\text { equation, } \\
\text { MUSLE, SCS } \\
\text { Curve } \\
\text { Number, } \\
\text { Yalin's } \\
\text { equation }\end{array}$ & $\begin{array}{l}\text { Erosion, } \\
\text { sedimentation, } \\
\text { runoff, from } \\
\text { agricultural } \\
\text { area }\end{array}$ & $\begin{array}{l}\text { suitable only } \\
\text { for field scale, } \\
\text { low potential } \\
\text { for } \\
\text { GIS integration }\end{array}$ & Yes & Yes & Yes & No & [65] \\
\hline
\end{tabular}


Table 5. Cont

\begin{tabular}{|c|c|c|c|c|c|c|c|c|c|c|c|c|c|c|c|}
\hline \multirow{2}{*}{ Sr. No. } & \multirow{2}{*}{ Model } & \multirow{2}{*}{ Description } & \multirow{2}{*}{$\begin{array}{c}\text { Developer } \\
\text { /Year* }\end{array}$} & \multicolumn{2}{|c|}{ Scale } & \multicolumn{2}{|c|}{ Input } & \multirow{2}{*}{$\begin{array}{l}\text { Governing } \\
\text { Equations }\end{array}$} & \multirow{2}{*}{$\begin{array}{l}\text { Model } \\
\text { Capabilities }\end{array}$} & \multirow{2}{*}{$\begin{array}{l}\text { Model } \\
\text { Limitations }\end{array}$} & \multicolumn{3}{|c|}{$\begin{array}{c}\text { Overland } \\
\text { Sedimentation }\end{array}$} & \multirow{2}{*}{$\begin{array}{c}\text { Channel } \\
\text { Sedimentation } \\
\text { Generation }\end{array}$} & \multirow{2}{*}{ Source } \\
\hline & & & & Temporal & Spatial & Demand & Variables & & & & $\mathrm{G}^{*}$ & $\mathrm{~T}^{*}$ & $\mathrm{D}^{*}$ & & \\
\hline 7 & $\begin{array}{l}\text { EROSION- } \\
\text { 2D/3D }\end{array}$ & EROSION & [89] & Event & $\begin{array}{l}\text { Field / } \\
\text { small } \\
\text { catchment }\end{array}$ & High & $\begin{array}{l}\text { Climate, soil } \\
\text { characteristics, } \\
\text { topography }\end{array}$ & $\begin{array}{l}\text { Mass balance } \\
\text { equation }\end{array}$ & $\begin{array}{l}\text { Simulation of } \\
\text { erosion }\end{array}$ & $\begin{array}{l}\text { Requires } \\
\text { extensive } \\
\text { computational } \\
\text { efforts }\end{array}$ & Yes & Yes & Yes & Yes & ([90] \\
\hline 8 & EUROSEM & $\begin{array}{l}\text { European Soil } \\
\text { Erosion } \\
\text { Model }\end{array}$ & [91] & Event & Catchment & High & $\begin{array}{l}\text { Climate, soil } \\
\text { characteristics, } \\
\text { land } \\
\text { use, topography }\end{array}$ & $\begin{array}{l}\text { Dynamic } \\
\text { mass balance } \\
\text { equation }\end{array}$ & $\begin{array}{l}\text { Simulation of } \\
\text { erosion, } \\
\text { sediment } \\
\text { yield, } \\
\text { deposition } \\
\text { and runoff }\end{array}$ & $\begin{array}{l}\text { Lower accuracy } \\
\text { for large } \\
\text { catchments }\end{array}$ & Yes & Yes & Yes & No & [92] \\
\hline 9 & GUEST & $\begin{array}{l}\text { Griffith } \\
\text { University } \\
\text { Erosion } \\
\text { System } \\
\text { Template }\end{array}$ & [93] & $\begin{array}{l}\text { Steady } \\
\text { State }\end{array}$ & Plot & High & $\begin{array}{l}\text { Climate, } \\
\text { watershed soil } \\
\text { characteristics, } \\
\text { runoff, } \\
\text { topography }\end{array}$ & $\begin{array}{l}\text { Mass balance } \\
\text { equation, } \\
\text { Deposition } \\
\text { Equation, } \\
\text { Rose } \\
\text { equation }\end{array}$ & $\begin{array}{l}\text { Simulation of } \\
\text { runoff, sedi- } \\
\text { mentation }\end{array}$ & $\begin{array}{l}\text { Low potential } \\
\text { for GIS } \\
\text { integration, } \\
\text { high data } \\
\text { requirement }\end{array}$ & Yes & Yes & Yes & No & [94] \\
\hline 10 & IDEAL & $\begin{array}{l}\text { Integrated } \\
\text { Design and } \\
\text { Evaluation of } \\
\text { loading } \\
\text { Models }\end{array}$ & [95] & Event & Catchment & High & $\begin{array}{l}\text { Climate, soil } \\
\text { characteristics, } \\
\text { land } \\
\text { Use and land } \\
\text { cover }\end{array}$ & MUSLE & $\begin{array}{l}\text { Sedimentation } \\
\text { yield, erosion, }\end{array}$ & $\begin{array}{l}\text { Rely on single } \\
\text { storm } \\
\text { events }\end{array}$ & Yes & Yes & Yes & Yes & [95] \\
\hline 12 & KINEROS & $\begin{array}{l}\text { KINematic } \\
\text { runoff and } \\
\text { EROSion } \\
\text { model }\end{array}$ & [98] & Event & $\begin{array}{l}\text { Small } \\
\text { Catchment, } \\
\text { hillslope } \\
\text { areas }\end{array}$ & High & $\begin{array}{l}\text { Rainfall, soil, } \\
\text { topography, } \\
\text { land cover, } \\
\text { drainage } \\
\text { network and } \\
\text { channel } \\
\text { geometry }\end{array}$ & $\begin{array}{l}\text { Bennett Mass } \\
\text { balance } \\
\text { equation, } \\
\text { sediment } \\
\text { transport } \\
\text { approach, } \\
\text { Kinematic } \\
\text { wave } \\
\text { equations }\end{array}$ & $\begin{array}{l}\text { Erosion, } \\
\text { sediment } \\
\text { yield, peak } \\
\text { runoff rate, } \\
\text { runoff }\end{array}$ & $\begin{array}{l}\text { Runoff } \\
\text { estimations are } \\
\text { based on single } \\
\text { storm events } \\
\text { without } \\
\text { considering } \\
\text { sub-surface } \\
\text { flows }\end{array}$ & Yes & Yes & Yes & No & [99] \\
\hline 13 & LASCAM & $\begin{array}{l}\text { Large Scale } \\
\text { Catchment } \\
\text { Model }\end{array}$ & [100] & Daily & Catchment & High & $\begin{array}{l}\text { Climate, } \\
\text { Surface } \\
\text { topography, } \\
\text { DEM, } \\
\text { streamflow and } \\
\text { sediment data }\end{array}$ & USLE & $\begin{array}{l}\text { Erosion, } \\
\text { sediment } \\
\text { yield, } \\
\text { nutrients }\end{array}$ & $\begin{array}{l}\text { Uncertainties in } \\
\text { the model } \\
\text { outputs }\end{array}$ & Yes & Yes & Yes & Yes & [67] \\
\hline
\end{tabular}


Table 5. Cont

\begin{tabular}{|c|c|c|c|c|c|c|c|c|c|c|c|c|c|c|c|}
\hline \multirow{2}{*}{ Sr. No. } & \multirow{2}{*}{ Model } & \multirow{2}{*}{ Description } & \multirow{2}{*}{$\begin{array}{c}\text { Developer } \\
\text { /Year * }\end{array}$} & \multicolumn{2}{|c|}{ Scale } & \multicolumn{2}{|c|}{ Input } & \multirow{2}{*}{$\begin{array}{l}\text { Governing } \\
\text { Equations }\end{array}$} & \multirow{2}{*}{$\begin{array}{l}\text { Model } \\
\text { Capabilities }\end{array}$} & \multirow{2}{*}{$\begin{array}{l}\text { Model } \\
\text { Limitations }\end{array}$} & \multicolumn{3}{|c|}{$\begin{array}{c}\text { Overland } \\
\text { Sedimentation }\end{array}$} & \multirow{2}{*}{$\begin{array}{c}\text { Channel } \\
\text { Sedimentation } \\
\text { Generation }\end{array}$} & \multirow{2}{*}{ Source } \\
\hline & & & & Temporal & Spatial & Demand & Variables & & & & $\mathrm{G}^{*}$ & $\mathrm{~T}^{*}$ & $\mathrm{D}^{*}$ & & \\
\hline 14 & MEFIDIS & $\begin{array}{l}\text { Modelo de } \\
\text { ErosaoFIsico } \\
\text { e } \\
\text { DIStribuido }\end{array}$ & [101] & Event & $\begin{array}{l}\text { Field scale } \\
\text { and small } \\
\text { catchment }\end{array}$ & High & $\begin{array}{l}\text { Climate, } \\
\text { topography, } \\
\text { Surface } \\
\text { topography, } \\
\text { DEM, } \\
\text { catchment } \\
\text { characteristics, } \\
\text { streamflow and } \\
\text { sediment data }\end{array}$ & $\begin{array}{l}\text { Diffusive } \\
\text { wave } \\
\text { equation, } \\
\text { Foster } \\
\text { equation } \\
\text { Kinetic } \\
\text { rainfall } \\
\text { energy } \\
\text { equation, } \\
\text { sediment } \\
\text { transport } \\
\text { capacity } \\
\text { approach }\end{array}$ & $\begin{array}{l}\text { Erosion, } \\
\text { runoff }\end{array}$ & $\begin{array}{l}\text { Soil erosion } \\
\text { based on } \\
\text { extreme rainfall } \\
\text { events, low } \\
\text { potential for } \\
\text { GIS integration }\end{array}$ & Yes & Yes & Yes & Yes & [101] \\
\hline 15 & MEDALUS & $\begin{array}{l}\text { Mediterranean } \\
\text { Desertifica- } \\
\text { tion and Land } \\
\text { Use research } \\
\text { programme } \\
\text { Model }\end{array}$ & {$[17,102]$} & Event & $\begin{array}{l}\text { Field scale } \\
\text { and small } \\
\text { catchment }\end{array}$ & High & $\begin{array}{l}\text { Climate, soil, } \\
\text { Land cover/ } \\
\text { land use, } \\
\text { Topography }\end{array}$ & $\begin{array}{l}\text { Mass } \\
\text { momentum } \\
\text { approach }\end{array}$ & $\begin{array}{l}\text { Erosion, } \\
\text { impact of } \\
\text { land use } \\
\text { changes }\end{array}$ & $\begin{array}{l}\text { Rely only on } \\
\text { recent data for } \\
\text { inputs }\end{array}$ & Yes & Yes & Yes & Yes & [103] \\
\hline 16 & PERFECT & $\begin{array}{l}\text { Productivity, } \\
\text { Erosion and } \\
\text { Runoff, } \\
\text { Functions to } \\
\text { Evaluate } \\
\text { Conservation } \\
\text { Techniques }\end{array}$ & [104] & Daily & Field & High & $\begin{array}{l}\text { Climate, soil, } \\
\text { crop, } \\
\text { tillage }\end{array}$ & MUSLE & $\begin{array}{l}\text { Erosion, } \\
\text { runoff, crop } \\
\text { yield }\end{array}$ & $\begin{array}{l}\text { Detailed } \\
\text { information on } \\
\text { crop } \\
\text { management } \\
\text { and tillage } \\
\text { practices }\end{array}$ & No & No & No & No & [105] \\
\hline 17 & $\begin{array}{c}\text { PEPP- } \\
\text { HILLFLOW }\end{array}$ & $\begin{array}{l}\text { Process } \\
\text { orientated } \\
\text { Erosion } \\
\text { Prediction } \\
\text { Program }\end{array}$ & [106] & Event & $\begin{array}{l}\text { Field scale } \\
\text { and small } \\
\text { catchment }\end{array}$ & High & $\begin{array}{l}\text { Climate, soil } \\
\text { characteristics, } \\
\text { Land cover/ } \\
\text { land use, } \\
\text { Topography, } \\
\text { nutrients }\end{array}$ & $\begin{array}{l}\text { Sediment } \\
\text { continuity } \\
\text { equation, } \\
\text { Foster } \\
\text { equation, } \\
\text { Yang's unit } \\
\text { stream power } \\
\text { method }\end{array}$ & $\begin{array}{l}\text { Runoff, } \\
\text { Erosion }\end{array}$ & $\begin{array}{l}\text { Rely on single } \\
\text { storm } \\
\text { Event, intensive } \\
\text { data } \\
\text { requirement }\end{array}$ & Yes & Yes & Yes & Yes & [106] \\
\hline 18 & RUNOFF & RUNOFF & [87] & Event & $\begin{array}{c}\text { Small } \\
\text { Catchment }\end{array}$ & Low & $\begin{array}{l}\text { Rainfall, soil } \\
\text { characteristics, } \\
\text { topography, } \\
\text { land cover, } \\
\text { drainage } \\
\text { network and } \\
\text { channel } \\
\text { geometry }\end{array}$ & $\begin{array}{l}\text { Splash } \\
\text { erosion, flow } \\
\text { rate } \\
\text { equations }\end{array}$ & $\begin{array}{l}\text { Erosion, } \\
\text { runoff, } \\
\text { sediment } \\
\text { yield }\end{array}$ & $\begin{array}{l}\text { Uncertainties in } \\
\text { input parameter } \\
\text { estimations and } \\
\text { model } \\
\text { validation }\end{array}$ & Yes & Yes & Yes & No & [87] \\
\hline
\end{tabular}


Table 5. Cont

\begin{tabular}{|c|c|c|c|c|c|c|c|c|c|c|c|c|c|c|c|}
\hline \multirow{2}{*}{ Sr. No. } & \multirow{2}{*}{ Model } & \multirow[t]{2}{*}{ Description } & \multirow{2}{*}{$\begin{array}{c}\text { Developer } \\
\text { /Year* }\end{array}$} & \multicolumn{2}{|c|}{ Scale } & \multicolumn{2}{|c|}{ Input } & \multirow{2}{*}{$\begin{array}{l}\text { Governing } \\
\text { Equations }\end{array}$} & \multirow{2}{*}{$\begin{array}{l}\text { Model } \\
\text { Capabilities }\end{array}$} & \multirow{2}{*}{$\begin{array}{l}\text { Model } \\
\text { Limitations }\end{array}$} & \multicolumn{3}{|c|}{$\begin{array}{c}\text { Overland } \\
\text { Sedimentation }\end{array}$} & \multirow{2}{*}{$\begin{array}{c}\text { Channel } \\
\text { Sedimentation } \\
\text { Generation }\end{array}$} & \multirow{2}{*}{ Source } \\
\hline & & & & Temporal & Spatial & Demand & Variables & & & & $\mathrm{G}^{*}$ & $\mathrm{~T}^{*}$ & $\mathrm{D}^{*}$ & & \\
\hline 19 & PESERA & $\begin{array}{l}\text { Pan- } \\
\text { European Soil } \\
\text { Erosion Risk } \\
\text { Assessment }\end{array}$ & [107] & Annual & Regional & Medium & $\begin{array}{l}\text { Climate, soil } \\
\text { characteristics, } \\
\text { land } \\
\text { cover, } \\
\text { topography }\end{array}$ & $\begin{array}{l}\text { Mass and } \\
\text { momentum } \\
\text { balance } \\
\text { equations, }\end{array}$ & $\begin{array}{l}\text { Runoff, } \\
\text { erosion, } \\
\text { sediment } \\
\text { yield, crop } \\
\text { yield }\end{array}$ & $\begin{array}{l}\text { Flow routing is } \\
\text { not well } \\
\text { developed }\end{array}$ & Yes & Yes & Yes & No & [19] \\
\hline 20 & $\begin{array}{l}\text { SHE/ } \\
\text { SHESED }\end{array}$ & $\begin{array}{l}\text { Systeme Hy- } \\
\text { drologique } \\
\text { Europian/- } \\
\text { Systeme Hy- } \\
\text { drologique } \\
\text { Europian } \\
\text { Sediment }\end{array}$ & [46] & Event & $\begin{array}{l}\text { Hillslope to } \\
\text { Catchment }\end{array}$ & High & $\begin{array}{l}\text { Rainfall, soil } \\
\text { characteristics, } \\
\text { topography, } \\
\text { land cover }\end{array}$ & $\begin{array}{l}\text { Mass and } \\
\text { momentum } \\
\text { balance } \\
\text { equations, } \\
\text { Yalin's } \\
\text { equation }\end{array}$ & $\begin{array}{l}\text { Erosion, } \\
\text { sediment } \\
\text { transport, } \\
\text { sediment } \\
\text { yield }\end{array}$ & $\begin{array}{l}\text { No simulation } \\
\text { of gully erosion }\end{array}$ & Yes & Yes & Yes & No & [46] \\
\hline 21 & WEPP & $\begin{array}{l}\text { Water Erosion } \\
\text { Prediction } \\
\text { Project }\end{array}$ & [108] & Daily & $\begin{array}{l}\text { Hillslope to } \\
\text { Catchment }\end{array}$ & High & $\begin{array}{l}\text { Climate, soil, } \\
\text { topography, } \\
\text { land use, field } \\
\text { management } \\
\text { and cultural } \\
\text { practices, } \\
\text { channel } \\
\text { network }\end{array}$ & $\begin{array}{l}\text { Steady-state } \\
\text { sediment } \\
\text { continuity } \\
\text { equation, } \\
\text { Foster } \\
\text { equation }\end{array}$ & $\begin{array}{l}\text { Runoff, } \\
\text { erosion, } \\
\text { sediment } \\
\text { yield }\end{array}$ & $\begin{array}{l}\text { Large number } \\
\text { of input } \\
\text { parameters, } \\
\text { neglect the } \\
\text { simulation in } \\
\text { permanent } \\
\text { channels }\end{array}$ & Yes & Yes & Yes & Yes & [109] \\
\hline 22 & WESP & $\begin{array}{l}\text { Watershed } \\
\text { erosion } \\
\text { simulation } \\
\text { program }\end{array}$ & [110] & Event & $\begin{array}{c}\text { Small } \\
\text { Catchment }\end{array}$ & Medium & $\begin{array}{l}\text { Climate, soil, } \\
\text { topography, } \\
\text { channel } \\
\text { network }\end{array}$ & $\begin{array}{l}\text { Kinematic } \\
\text { wave } \\
\text { equations, }\end{array}$ & $\begin{array}{l}\text { Simulation of } \\
\text { runoff and } \\
\text { erosion * }\end{array}$ & $\begin{array}{l}\text { Intensive } \\
\text { computation of } \\
\text { input } \\
\text { parameters }\end{array}$ & Yes & Yes & Yes & Yes & [110] \\
\hline 23 & $\begin{array}{l}\text { WATEM/ } \\
\text { SEDEM }\end{array}$ & $\begin{array}{l}\text { Water and } \\
\text { Tillage } \\
\text { Erosion } \\
\text { Model/Sediment } \\
\text { Delivery } \\
\text { Model }\end{array}$ & [111] & Annual & Field & Low & $\begin{array}{l}\text { Climate, soil } \\
\text { characteristics, } \\
\text { land } \\
\text { cover, flow } \\
\text { network }\end{array}$ & RUSLE & $\begin{array}{l}\text { Erosion, } \\
\text { tillage } \\
\text { erosion, } \\
\text { sedimentation }\end{array}$ & $\begin{array}{l}\text { Require high } \\
\text { quality detailed } \\
\text { watershed } \\
\text { information }\end{array}$ & Yes & Yes & Yes & Yes & [112] \\
\hline 24 & SEMMED & $\begin{array}{l}\text { Soil Erosion } \\
\text { Model for } \\
\text { Mediterranean } \\
\text { Areas }\end{array}$ & [112] & Annual & $\begin{array}{l}\text { Regional } \\
\text { scale }\end{array}$ & Medium & $\begin{array}{l}\text { DEM, climate, } \\
\text { soil } \\
\text { characteristics, } \\
\text { channel } \\
\text { network and } \\
\text { geometry }\end{array}$ & $\begin{array}{l}\text { Distributed } \\
\text { transport } \\
\text { capacity }\end{array}$ & $\begin{array}{l}\text { Simulate the } \\
\text { distributed } \\
\text { character of } \\
\text { the erosion } \\
\text { process, } \\
\text { predicts soil } \\
\text { loss }\end{array}$ & $\begin{array}{l}\text { Sensitive to } \\
\text { storage capacity, } \\
\text { soil moisture, } \\
\text { soil } \\
\text { detachability } \\
\text { index }\end{array}$ & Yes & Yes & Yes & No & [113] \\
\hline 25 & SIMWE & $\begin{array}{l}\text { Simulation of } \\
\text { Water Erosion }\end{array}$ & [34] & Event & Catchment & High & $\begin{array}{l}\text { Rainfalls, } \\
\text { surface } \\
\text { roughness, } \\
\text { DEM }\end{array}$ & $\begin{array}{l}\text { Saint Venant } \\
\text { equation for } \\
\text { continuity of } \\
\text { flow, } \\
\text { Manning's n } \\
\text { value. }\end{array}$ & $\begin{array}{l}\text { Erosion, gully } \\
\text { Formation, } \\
\text { sediment } \\
\text { transport and } \\
\text { deposition }{ }^{*}\end{array}$ & $\begin{array}{l}\text { Require high } \\
\text { quality detailed } \\
\text { watershed } \\
\text { information }\end{array}$ & Yes & Yes & Yes & No & [114] \\
\hline
\end{tabular}


Table 5. Cont.

\begin{tabular}{|c|c|c|c|c|c|c|c|c|c|c|c|c|c|c|c|}
\hline \multirow{2}{*}{ Sr. No. } & \multirow{2}{*}{ Model } & \multirow[t]{2}{*}{ Description } & \multirow{2}{*}{$\begin{array}{l}\text { Developer } \\
\text { /Year* }\end{array}$} & \multicolumn{2}{|c|}{ Scale } & \multicolumn{2}{|c|}{ Input } & \multirow{2}{*}{$\begin{array}{l}\text { Governing } \\
\text { Equations }\end{array}$} & \multirow{2}{*}{$\begin{array}{l}\text { Model } \\
\text { Capabilities }\end{array}$} & \multirow{2}{*}{$\begin{array}{l}\text { Model } \\
\text { Limitations }\end{array}$} & \multicolumn{3}{|c|}{$\begin{array}{c}\text { Overland } \\
\text { Sedimentation }\end{array}$} & \multirow{2}{*}{$\begin{array}{c}\text { Channel } \\
\text { Sedimentation } \\
\text { Generation }\end{array}$} & \multirow{2}{*}{ Source } \\
\hline & & & & Temporal & Spatial & Demand & Variables & & & & $\mathrm{G}^{*}$ & $T^{*}$ & $D^{*}$ & & \\
\hline 26 & RHEM & $\begin{array}{l}\text { Rangeland } \\
\text { Hydrology } \\
\text { and Erosion } \\
\text { Model }\end{array}$ & [115] & Event & $\begin{array}{l}\text { Field scale } \\
\text { and small } \\
\text { catchment }\end{array}$ & High & $\begin{array}{l}\text { Climate, soil } \\
\text { characteristics, } \\
\text { watershed } \\
\text { characteristics }\end{array}$ & $\begin{array}{l}\text { Sediment } \\
\text { transport } \\
\text { equation }\end{array}$ & $\begin{array}{l}\text { uRnoff, } \\
\text { erosion, } \\
\text { sediment } \\
\text { yield }\end{array}$ & $\begin{array}{l}\text { less suitable for } \\
\text { simulation of } \\
\text { rangeland } \\
\text { surfaces }\end{array}$ & Yes & Yes & Yes & No & [116] \\
\hline 27 & TOPOG & TOPOG & [117] & Daily & $\begin{array}{l}\text { Hillslope to } \\
\text { Catchment }\end{array}$ & High & $\begin{array}{l}\text { Climate, soil, } \\
\text { topography, } \\
\text { Land cover }\end{array}$ & $\begin{array}{l}\text { Equations for } \\
\text { sediment } \\
\text { transport in } \\
\text { channels }\end{array}$ & Erosion & $\begin{array}{l}\text { Extensive input } \\
\text { data } \\
\text { requirements } \\
\text { and a high } \\
\text { number of } \\
\text { physical } \\
\text { parameters } \\
\text { (complex) }\end{array}$ & Yes & Yes & Yes & No & [118] \\
\hline
\end{tabular}

* additional model capabilities besides soil erosion; G: Generation; T: Transportation; D: Deposition; Developer/year: references for model manuals and first research articles describing respective model. 


\subsubsection{Remote Sensing (RS) and GIS-based Soil Erosion Modeling}

Remote Sensing data combined with GIS tools provide the powerful capabilities for mapping soil characteristics and soil resources over high spatial and temporal resolution in a timely and cost-effective way [119]. Soil erosion models can be incorporated into GIS tools and combined with RS data. RS derived climate data, land use/land cover information, and their integration with GIS can be used for soil erosion modeling [120]. Remote sensing based digital elevation/terrain model (DEM/DTM) is an important tool to provide inputs to the soil erosion models, catchment rainfall/runoff relationship development, and sedimentation processes [121-123]. Various GIS techniques (QGIS, ArcGIS) use Digital Elevation Models (DEM) and can derive multiple variables for topographical parameterization such as slope, aspect ratio, drainage, stream and catchment delineation, surface flow, and soil erodibility factor [124].

Many well-known soil erosion models i.e., USLE (Universal Soil Loss Equation, 1965) [3], RUSLE (Revised Universal Soil Loss Equation, 1997) [125], SEMMED (Soil Erosion Model for Mediterranean Regions, 1999) [113], PESERA (Pan-European Soil Erosion Risk Assessment, 2003) [37], EUROSEM (European Soil Erosion Model, 1993) [126], and EGEM (Ephemeral Gully Erosion Model, 1999) [86], integrated with RS and GIS techniques, have been widely used.

The use of GIS and RS for soil erosion and sedimentation modeling may involve certain consequences including multiple data sources based on vast data requirements, computing expertise for model re-scaling and data reliability issues, and complex verification algorithms of model outputs [127].

\subsection{Description of Selected Models with Respect to Plot Scale Simulations}

A list of different soil erosion models is presented in Section 3.3. These models vary in their range of complexity, data requirements, the scale of application, and key limitations. This section aims to provide a brief introduction to models selected on their applicability to a plot/field scale. The shortlisted models are reviewed in terms of their objective, model structure, components, and their assimilation, and model calibration ease and parameter requirements are presented in this section. The review of models is limited to those models with strict consideration of soil erosion generation at a plot or field scale. Therefore, many other commonly applied models, for example SWAT, EUROSEM, etc., are not discussed in this section (Table 6). In order to assess the model capabilities to simulate soil erosion, application examples of selected models in different climatic zones were reviewed and summarized in Table 6 . The Nash-Sutcliffe efficiency method was the most commonly used method for evaluation of model performance. Further, Root mean square error, coefficient correlation, average absolute error, and coefficient of determination were commonly applied measures.

Most studies report a sensitivity of simulated sediment deposition to different environmental and management factors, such as rainfall, crop management factors, soil physical properties, and vegetation cover. Selected models were tested at field or plot scales under different cropping systems and field conditions. Calibration of field scale models based on data from fields, that are characterized by a high spatial heterogeneity of topography and soil types, is more accurate than using spatial averaged data from larger catchment areas. Most of the field scale models are based on one-dimensional equations (Saint Venant equation or Kinematic wave theory) for estimation of overland flows, thereby limiting their capabilities for spatially distributed modeling. Only a few models, such as WEPP, TCRP, and CREAMS, also represent the water movement through the unsaturated part of the soil profile which influences the runoff on hillslopes (Table 6). 
Table 6. Examples of applications of some selected field scale soil erosion models.

\begin{tabular}{|c|c|c|c|c|c|c|c|c|c|c|c|}
\hline Sr. No. & Model & Description & $\begin{array}{c}\text { Spatial } \\
\text { Dimension }\end{array}$ & Model Type & Study Area & Objective & $\begin{array}{l}\text { Input Data } \\
\text { Used }\end{array}$ & $\begin{array}{l}\text { Method Used } \\
\text { for Evaluation }\end{array}$ & Conclusion & Remarks & Reference \\
\hline 1 & EPM & $\begin{array}{l}\text { Erosion } \\
\text { Potential } \\
\text { Method }\end{array}$ & $1 \mathrm{D}$ & Dynamic & $\begin{array}{l}\text { Alfenas } \\
\text { Municipality, } \\
\text { (437 ha) }\end{array}$ & $\begin{array}{l}\text { Simulation of } \\
\text { surface runoff, } \\
\text { soil erosion, } \\
\text { comparing } \\
\text { results with } \\
\text { RUSLE of SLT }\end{array}$ & $\begin{array}{l}\text { DEM, climate } \\
\text { data, soil } \\
\text { characteristics }\end{array}$ & $\begin{array}{l}\text { Sediment } \\
\text { retention } \\
\text { coefficient, } \\
\text { RMSE, } \\
\text { correlation- } \\
\text { coefficient }\end{array}$ & $\begin{array}{l}\text { Correlations of the } \\
\text { potential values of soil } \\
\text { erosion between EPM } \\
\text { and RUSLE showed a } \\
\text { similar pattern for the } \\
\text { different land } \\
\text { management types and } \\
\text { land uses despite the } \\
\text { different orders of } \\
\text { magnitude }\end{array}$ & $\begin{array}{l}\text { For calibration, } \\
\text { EPM requires } \\
\text { experimental } \\
\text { validation, } \\
\text { which would be } \\
\text { subjected to } \\
\text { heterogeneity of } \\
\text { crop and soil in } \\
\text { the field }\end{array}$ & [128] \\
\hline 2 & TCRP & $\begin{array}{l}\text { Tillage- } \\
\text { Controlled } \\
\text { Runoff Pattern } \\
\text { model }\end{array}$ & $2 \mathrm{D}$ & Dynamic & Multiple sites & $\begin{array}{l}\text { Sediment } \\
\text { fluxes, } \\
\text { deposition } \\
\text { processes in a } \\
\text { 2-D spatial } \\
\text { context }\end{array}$ & $\begin{array}{l}\text { sediment } \\
\text { deposition } \\
\text { equations }\end{array}$ & $\begin{array}{l}\text { Correlation } \\
\text { coefficient }\end{array}$ & $\begin{array}{l}\text { Model is capable of } \\
\text { simulating both spatial } \\
\text { pattern and size } \\
\text { selectivity of deposition } \\
\text { pattern in tilled fields }\end{array}$ & $\begin{array}{l}\text { Understanding } \\
\text { and } \\
\text { representation of } \\
\text { sediment } \\
\text { delivery and } \\
\text { deposition need } \\
\text { to be improved } \\
\end{array}$ & [129] \\
\hline 3 & WEPP & $\begin{array}{l}\text { Watershed } \\
\text { Erosion } \\
\text { Prediction } \\
\text { Project }\end{array}$ & $2 \mathrm{D}$ & Dynamic & $\begin{array}{l}\text { Demonstration } \\
\text { farm, } \\
\text { Ratchaburi } \\
\text { province, } \\
\text { Thailand }\end{array}$ & $\begin{array}{l}\text { Performances } \\
\text { of the WEPP } \\
\text { under } \\
\text { conservation } \\
\text { cropping } \\
\text { system }\end{array}$ & $\begin{array}{l}\text { Monthly } \\
\text { rainfall, Land } \\
\text { use map, Soil } \\
\text { map, DEM, } \\
\text { Daily } \\
\text { Sediment }\end{array}$ & NSE & $\begin{array}{l}\text { WEPP model predicted } \\
\text { lower values of runoff } \\
\text { and sediment yield. } \\
\text { WEPP coupled with } \\
\text { MIKE SHE/MIKE } 11 \\
\text { capable to simulate soil } \\
\text { losses in different } \\
\text { conservation practices }\end{array}$ & $\begin{array}{l}\text { Satisfactory } \\
\text { Performance for } \\
\text { sediment yield } \\
\text { estimation at } \\
\text { small scale }\end{array}$ & [130] \\
\hline 4 & APSIM & $\begin{array}{l}\text { Agricultural } \\
\text { Production } \\
\text { Simulation }\end{array}$ & $1 \mathrm{D}$ & Dynamic & $\begin{array}{l}16 \text { plots, } 52 \mathrm{~m}^{2} \\
(4 \mathrm{~m} \times 13 \mathrm{~m}) \\
\text { in area each } \\
\text { plot }\end{array}$ & $\begin{array}{l}\text { Modeling } \\
\text { effects of } \\
\text { tillage on soil } \\
\text { water } \\
\text { dynamics }\end{array}$ & $\begin{array}{l}\text { Daily } \\
\text { temperature, } \\
\text { daily rainfall, } \\
\text { Tree zoning }\end{array}$ & $\mathrm{R}^{2}, \mathrm{NSE}, \mathrm{RSR}$ & $\begin{array}{l}\text { APSIM is adequate for } \\
\text { agroforestry system }\end{array}$ & $\begin{array}{l}\text { APSIM requires } \\
\text { modification in } \\
\text { soil erosion } \\
\text { component }\end{array}$ & [131] \\
\hline 5 & EPIC & $\begin{array}{l}\text { Erosion- } \\
\text { Productivity } \\
\text { Impact } \\
\text { Calculator }\end{array}$ & $1 \mathrm{D}$ & Dynamic & $\begin{array}{l}\text { South-central } \\
\text { Chile }\end{array}$ & $\begin{array}{l}\text { Simulation of } \\
\text { soil erosion }\end{array}$ & $\begin{array}{l}\text { DEM, climate } \\
\text { data, soil } \\
\text { characteristics }\end{array}$ & $\begin{array}{l}\text { correlation } \\
\text { coefficient, } \\
\text { RMSE }\end{array}$ & $\begin{array}{l}\text { Calculated rates of soil } \\
\text { erosion was } \\
\text { overestimated as slope } \\
\text { segment is relatively } \\
\text { difficult to decide }\end{array}$ & $\begin{array}{l}\text { EPIC predicts } \\
\text { two times more } \\
\text { soil erosion } \\
\text { under wheat and } \\
\text { conventional } \\
\text { tillage } \\
\text { comparing to } \\
\text { WEPP and USLE }\end{array}$ & [132] \\
\hline
\end{tabular}


Table 6. Cont

\begin{tabular}{|c|c|c|c|c|c|c|c|c|c|c|c|}
\hline Sr. No. & Model & Description & $\begin{array}{c}\text { Spatial } \\
\text { Dimension }\end{array}$ & Model Type & Study Area & Objective & $\begin{array}{l}\text { Input Data } \\
\text { Used }\end{array}$ & $\begin{array}{l}\text { Method Used } \\
\text { for Evaluation }\end{array}$ & Conclusion & Remarks & Reference \\
\hline 6 & CREAMS & $\begin{array}{l}\text { Chemicals, } \\
\text { Runoff and } \\
\text { Erosion from } \\
\text { Agricultural } \\
\text { Management } \\
\text { Systems }\end{array}$ & $2 \mathrm{D}$ & Dynamic & Finland & $\begin{array}{l}\text { Predicting } \\
\text { field-scale } \\
\text { runoff } \\
\text { and erosion, } \\
\text { modify the } \\
\text { model for } \\
\text { Finnish } \\
\text { conditions }\end{array}$ & $\begin{array}{l}\text { Mean daily } \\
\text { temperatures } \\
\text { and rainfall, } \\
\text { Evapotranspi- } \\
\text { ration, surface } \\
\text { albedo, leaf } \\
\text { area index, }\end{array}$ & $\begin{array}{l}\text { AERR, RMSE, } \\
\text { NSE }\end{array}$ & $\begin{array}{l}\text { Snow accumulation and } \\
\text { snowmelt description, } \\
\text { adjustable albedo } \\
\text { introduction into } \\
\text { CREAMS improved } \\
\text { simulations of runoff } \\
\text { volumes }\end{array}$ & $\begin{array}{l}\text { SCS curve } \\
\text { number can be } \\
\text { introduced for } \\
\text { more physically } \\
\text { based } \\
\text { representation of } \\
\text { runoff in alley } \\
\text { cropping system }\end{array}$ & [133] \\
\hline 8 & PERFECT & $\begin{array}{l}\text { The } \\
\text { Productivity, } \\
\text { Erosion and } \\
\text { Runoff, } \\
\text { Functions to } \\
\text { Evaluate } \\
\text { Conservation } \\
\text { Techniques }\end{array}$ & $1 \mathrm{D}$ & Dynamic & $\begin{array}{l}\text { Plot scale, } \\
\text { Queensland }\end{array}$ & $\begin{array}{l}\text { Simulates } \\
\text { interactions } \\
\text { between soil } \\
\text { type, climate, } \\
\text { and fallow } \\
\text { management } \\
\text { strategy and } \\
\text { crop sequence. }\end{array}$ & $\begin{array}{l}\text { Initial soil } \\
\text { moisture, soil } \\
\text { characteristics, } \\
\text { topography, } \\
\text { Landuse }\end{array}$ & $\mathrm{R}^{2}$ & $\begin{array}{l}\text { PERFECT does not } \\
\text { consider rainfall intensity } \\
\text { and represents less } \\
\text { accurate soil erosion on } \\
\text { daily time steps. }\end{array}$ & $\begin{array}{l}\text { The validated } \\
\text { model can be } \\
\text { coupled with } \\
\text { soil and } \\
\text { long-term } \\
\text { climate } \\
\text { databases to } \\
\text { simulate } \\
\text { probabilities of } \\
\text { production and } \\
\text { erosion risks due } \\
\text { to climatic } \\
\text { variability. }\end{array}$ & [135] \\
\hline
\end{tabular}

R2: Coefficient of determination, NSE: Nash-Sutcliffe efficiency, RSR: RMSE-observations standard deviation ratio, RMSE: Root Mean Square Error, ME: Model efficiency, AERR: average absolute error. 


\subsubsection{Erosion Potential Method, EPM}

Erosion Potential Method (EPM) is an empirical model to simulate water erosion from fields to small catchments, using input data related to meteorology and the matrix of the catchment physical characteristics. The model has been widely applied worldwide. It contains an advanced classification procedure using four characteristics including erosion coefficient, land use coefficient, soil erodibility, and mean slope in different land units [32]. Kouhpeima et al. [136] state that EPM is a method for easy and rapid analysis of erosion risk and sedimentation. The accuracy of results depends on the values of erosion coefficients. Moreover, EPM considers only four factors for erosion assessment and can be applied to small areas where database layers are limited. EPM integration with GIS and remote sensing could be a useful technique in the identification of soil loss and sedimentation in areas with insufficient sediment gauging stations [137]. The over-/under-prediction limits of EPM simulations are within 13 percent from the measured values and are considered to have acceptable accuracy for soil loss simulations at the catchment scale $[138,139]$.

\subsubsection{Tillage-Controlled Runoff Pattern Model, TCRP}

The TCRP model has been evaluated in different environments globally, mainly for the prediction of runoff patterns with the flow along the direction of plow lines in tilled fields within a catchment. This model requires a digital elevation model, land use maps, and tillage orientation as inputs. The model creates a tillage-controlled runoff pattern along with a topographic controlled runoff pattern. The use of the first one in event-based deterministic models results in a much better level of accuracy for runoff and erosion patterns with field observations. Model simulations show that tillage information should be included when estimating runoff directions if erosion pattern accuracy is under question. Souchere et al. [140] proposed that to analyze the tillage impact on runoff in a spatially distributed water model, each cell must be assigned to a tillage direction. This results in complexities as flow lines may cross each other and ditches may exist on the field. Souchere et al. [141] solved these problems by changing the runoff direction manually and assuming the runoff is always in the direction of tillage. However, it would be laborious for large catchments to be modeled.

The TCRP model was developed using raster language to specifically integrate with the GIS tool [142]. The model requires surface physical characteristics, DEM, land use maps, and tillage direction information as inputs. The maps should have an area larger than that of the catchment to be modeled because catchment boundaries can be defined after only the assessment of runoff patterns.

\subsubsection{Soil Loss Estimation Model for Southern Africa, SLEMSA}

SLEMSA was developed by [35] in Zimbabwe as a framework for estimating local soil losses by using details of local environmental conditions driving soil erosion process i.e., climate, soil types, topography, soil cover, and field management practices $[143,144]$. The SLEMSA modeling approach consists of four major steps: (1) identify major control variables (rainfall energy, interception, etc.), for which the values are easily measured and have a rational physical explanation, (2) develop a relationship, called submodels, between selected variables and soil losses, (3) formulate the model to relate these submodels, (4) test the model [143]. Heydarnejad et al. [130] examined SLEMSA in series of tests, with careful monitoring of controlled variables on selected plots; errors of 9 to 18 percent were noted. SLEMSA claims to be simpler relatively to the USLE as it is less data demanding with high extrapolation capabilities [24]. GIS can be used to calculate SLEMSA control variables that upon formulation provide potential soil losses within the catchment [145]. A study of SLEMSA in mountainous terrain by [146] indicates the sensitivity of potential soil loss to both slope steepness and rainfall erosivity resulting in an overestimation of soil loss with steep slopes and high rainfall intensities [28]. 


\subsubsection{Agricultural Production Simulation, APSIM}

APSIM, a dynamic conceptual modeling platform, was developed by the Agricultural Production Systems Research System Unit (APSRU) in Queensland. APSIM modeling platform has been evaluated worldwide in different environmental conditions ranging from interpretation of on-farm experiments to risk assessment of a range of alternative management options [147] mainly to simulate the crop production in relation to climate, soil erosion, and field management practices while identifying long-term solutions for natural resource management issues at field scale using input data provided at daily time steps $[50,148]$. Since APSIM offers many modules (generally categorized as biological and environmental modules), the erosion model is capable to simulate the impact of erosion on the soil profile as soil loss occurs. The erosion module remains unaware of the impact of other modules on the profile. The estimation of daily soil loss is performed by either of two submodels (1) Freebairn and (2) Rose [50]. The lateral one uses the USLE equation $[104,149]$. The module was revised to consider runoff and land cover, which can be affected by management within the APSIM model. The soil erosion measurements required for calibration are based on the runoff volume, soil cover, soil erodibility, and slope-length factor along with management practices. A different module within APSIM provides the values of these factors i.e., the surface cover is provided by the soil organic matter module, and the SWIM module provides surface runoff. Basche et al. [51] have successfully calibrated and validated the APSIM model to predict runoff and sediment yield. Further, the tested APSIM model was implemented for soil loss based risk management and supporting practices. However, the use of this model is only recommended when sufficient data is available. Notably, APSIM has high input demand; most uses require extensive field investigations.

\subsubsection{RillGrow}

The RillGrow model is capable to predict a realistic spatial pattern of the rill network in response to a given rainfall event [52]. The erosion model series of RillGrow mainly expresses the eroding hillslopes on a small scale as a self-organized dynamical system producing a rill network [150]. Digital elevation models of the hillslopes used as an input to the RillGrow simulates the rill network as a whole system which later on is compared with the field and laboratory experiments for validation [151]. A logistic S-Curve, the relationship between flow energy and sediment load, is considered to estimate erosion resulting from the surface flow. Hillslope micro-topography could be responsible for the observed vitality of rill competition and spatial pattern of overland flow initiating lowering of the surface. Such modifications change the path of the soil erosion process as it creates its own surface [152]. This simple relationship develops a complex rill network. However, this simplicity results in limited model computational abilities as the flow process and erosion have to be predicted on a microscale. Also, model data requirement creates issues. These limitations make this model impractical for real-world erosion simulations. Simulations have an immense demand of computation time if the area is larger than the hillslope plot or laboratory experiment [153]. There are a few articles on GIS integration with the RillGrow model in the latest versions. That needs to be worked out to improve the workability of the RillGrow model in the future.

\subsubsection{Erosion-Productivity Impact Calculator, EPIC}

EPIC is a detailed model developed to simulate, simultaneously and realistically, the physical processes involved by developing the relationship between soil losses and soil productivity. EPIC mainly uses climate, land cover, tillage, and soil characteristics as input variables. Many applications of EPIC have been studied in the United States and worldwide under varying environmental conditions, for example, climate change effects on crop yield and soil erosion [154,155], wind erosion [156], irrigation impacts on crop yields [157,158], assessment of soil temperature [159], and soil carbon sequestration as a function of management and cropping systems $[160,161]$. The model has been 
extensively tested in many ways. The EPIC model has nine major components, namely, weather, nutrients, plant growth, soil temperature, hydrology, environment, and economics. Each component was tested $[65,88,162-165]$ and the results were found to be acceptable and reliable. [166] proposes that the EPIC model works more efficiently over small areal extent (generally $\sim 1$ ha) because management practices and soils are considered homogenous. However, the model can consider all kinds of soil properties. Traditionally, EPIC is site-specific, but when integrated with GIS tools, regional crop growth and yield can be simulated (e.g. the G-EPIC version [166]. GIS is used to produce model inputs for DEM, land use/land cover, and soil maps. Due to EPIC's extensive testing and high integration with GIS, its application has been increased and has become famous among scientists.

\subsubsection{Chemicals, Runoff, and Erosion from Agricultural Management System, CREAMS}

The Chemicals, Runoff, and Erosion from Agricultural Management System model was developed in the United States [167]. CREAMS is a physical, daily-based dynamic model that simulates runoff, erosion, and sediment yield, having a capacity for assessment of nutrient loss and chemicals from agricultural lands suitable at field scale [65]. Hydrology is one of the components of CREAMS that is the principal element to simulate soil erosion neglecting the deep percolation. With the daily rainfall data, the SCS curve number is used to estimate surface runoff [168]. These component results provide the input to other components of CREAMS to estimate nutrient and chemical losses. The erosion component in CREAMS uses USLE along with sediment transport for overland flows. Studies show that the CREAMS model performs better for field-scale but it can be applied to larger-scales ( $400 \mathrm{ha})$ [5]. This may be due to the fact that the model assumes uniform topography and land use and it does not consider temporal variations in soil erodibility which is highly unrealistic in the real world. Moreover, Govers et al. [169] observed that dynamic simulation of water erosion may limit the accuracy of estimations because of their extensive dependents on the validated input data. However, such limits can be reduced using physically based models rather than empirical models such as CREAMS.

\subsubsection{The Griffith University Erosion System Template, GUEST}

The Griffith University Erosion System Template [93] is a physically based steady-state sediment flux model developed to simulate single events of erosion resulting in temporal variations in sediment yields at a plot scale. The model uses hydrological and surface characteristics of uniform slope and relates rainfall-runoff rates to predict the yield of eroded sediments [94]. The model algorithms explaining erosion, transport, and sediment yield are based on single rainfall events at the plot scale. GUEST considers the erosion process to mainly be due to rainfall impact and the effect of overland flow generated shear stresses exerted on soil, making GUEST a comparatively complex process-based model which requires a large number of input data. Huang et al. [170] noted the low accuracy in predicted soil erosion by surface runoff when applied to the catchment scale and that it was limited by the extent of data required at the plot scale.

\subsubsection{The Productivity, Erosion and Runoff, Functions to Evaluate} ConservationTechniques, PERFECT

The PERFECT model [105] was developed by the Queensland Department of Primary Industries and the QDPI/CSIRO Agricultural Production System Research Unit in Australia. This model was developed to integrate with other physically based models such as CREAMS for studying the impact of soil management factors i.e., field preparation practices and soil conservation techniques. CREAMS excludes the land cover variations caused by tillage practices to estimate surface runoff. PERFECT model considers management strategies to predict surface runoff, erosion (MUSLE), and crop production on daily time steps at the field scale. As other models can be incorporated into the PERFECT model, it is a mix of conceptual, empirical, and physically based models.

Littleboy et al. [171] suggested that the PERFECT model is more accurate than CREAMS for estimating runoff, accounting for $77-89 \%$ of the variation in measured [172] 
daily runoff volume. However, this model does not consider the impact of rainfall intensity thus resulting into over/underestimation of soil erosion based on a single rainfall event. Sediment and nutrient components may be added for water quality modeling that may provide an advantage with crop cover data and management components where it is needed.

\section{Discussion}

\subsection{Selection Criteria for Soil Erosion Models}

Each soil erosion model has its predictive capabilities and modeling processes and its applicability depends on its intended use, available input and calibration data, temporal and spatial scale, and required accuracies. Based on the review work, the selection of a suitable model for a distinct purpose at the field-scale should be guided by the following criteria. (1) Problem recognition: Define the problem statement in a clear way to achieve a maximum match between the problem to be solved and the model objectives. (2) Spatial scale: the next criteria is to decide whether the model is compatible with the plot or field scale. (3) Data availability: make a list of required input data (topography, climate, field investigations) and their availability. (4) Temporal scales to be considered (event-based or continuous) (5) Elements to be assessed; decide which elements of the catchment are to be modeled i.e., overland erosion and sedimentation, hillslope erosion, or channel/stream erosion and sedimentation. (6) Model sensitivity; the uncertainties within input data should be identified that may impact the reliability of the simulated results before the model evaluation. (7) Model validation; simulation results must be compared with field observations that may also use for model calibration before the simulation process.

\subsection{Capabilities and Limitations of Field Scale Models}

Soil erosion models are bound to have certain strengths and limitations depending on their different development objectives and often specific environmental processes and conditions. Most of the available soil erosion models have been developed mainly for larger scales (basin or watershed) where spatial variations in soil conditions (soil erodibility, soil cover, slope, and tillage practices) and hydrological conditions (surface runoff, infiltration rate, and rainfall intensity) are significant compared to those at small scales (field or plot). Individual hydro-geomorphological processes and vegetation impact differently on soil erosion process across various scales. Slope arguably is one of the major factors in the erosion process. For models such as EPIC, WEPP, CREAMS, and GLEAMS that use USLE to reflect the effect of slope length on soil erosion, the major problem (Table 6) is the suitable selection of slope segments in fields with complex topography where slope characteristics may vary drastically. EUROSEM is a model that uses a dynamic mass balance equation to simulate the erosion process at a field scale for agricultural lands. EPIC predicts soil losses from rill and inter-rill areas all together whereas EUROSEM, WEPP, and GLEAMS estimate each separately.

The assumptions regarding the soil erodibility and tillage formation have a great influence on predicting the volume and direction of surface runoff and subsequently soil losses. TCRP is a 2-D empirical model, which simulates runoff patterns with the flow along the direction of plow lines in tilled fields assuming runoff direction always in direction of tillage (Table 6). TCRP can consider both tillage-controlled runoff patterns and topographycontrolled runoff patterns. EPIC runoff factor considers ridge heights between furrows to estimate total runoff. EUROSEM and WEPP coupled with MIKE SHE/MIKE 11 can simulate daily soil losses considering different conservation practices at the field scale.

Although the above mentioned problems are significant, calibration of field scale models in fields characterized by spatial heterogeneity of topography and soil is more accurate than for larger catchment areas. Furthermore, the accuracy of the simulation of erosion rates depends on the spatial dimension taken into account, i.e. whether processes are simulated at the soil profile scale (1D, point based assuming a field with homogeneous soil and terrain conditions) and/or spatially distributed method (2D/3D) (Table 6). The 
quality and accuracy of the calibration of the erosion processes in heterogeneous fields should increase with the dimension that is considered. However, a major bottleneck for the multi-dimensional models is the availability and accuracy of soil information. On the other hand, the accuracy and availability of topographic information has considerably improved in the last decade (e.g. radar and laser based sensors carried by UAV or airplanes).

\subsection{Model Comparison with Respect to Simulating Soil Erosion in Complex Cropping Systems}

Conservation practices like strip and patch cropping or agroforestry systems are important management options to improve floristic and faunistic diversity in intensively used agricultural landscapes. Tools are required to predict the impacts of such diverse cropping systems on soil erosion processes. The Water Erosion Prediction Project (WEPP) for the intercropping system, Water Nutrient and Light Capture in Agroforestry Systems (WaNuLCAS), and Agricultural Production Simulation (APSIM) models are capable of simulating soil erosion in conventional and complex cropping systems at the field scale. WaNuLCAS represents dynamic processes in the spatial domain. It was designed to simulate Tree-SoilCrop interactions under a wide range of agroforestry systems. It uses the Rose equation [40] to simulate the erosion process in a simplified 2D approach. However, this component of WaNuLCAS has not been tested extensively and requires further investigation. APSIM offers many modules (generally categorized as biological and environmental modules). The erosion model is capable of simulating the impact of erosion on the soil profile as soil loss occurs under different management practice options such as strip cropping and alley cropping systems, but it considers only one dimension in the field. Notably, APSIM has high input demand, most of which requires extensive field investigations.

Another important aspect is the translation of rainfall to runoff under varying canopy interception in complex cropping systems. A realistic representation of the role of canopy cover in rainfall-runoff modeling is essential when predicting sediment transport in heterogeneous fields along hillslopes. A few models such as EPIC, WEPP, EUROSEM, CREAMS, SCUAF, and WaNuLCAS account for the intercepted rainfall to estimate total runoff (Table 6). However, to this date, there is no cropping model available at the field scale that considers the impact of cropping systems like strip cropping and patch cropping and their complex canopy arrangements affecting runoff induced soil erosion processes. EPIC, WEPP, EUROSEM, and CREAMS models may have the capabilities to model complex variations in cropping systems when integrated with GIS [11,84,88].

The review suggests that further studies have to be conducted to develop tools that facilitate the integration of modeling components to lower the complexities of source codes and to further improve existing models or develop new models that represent soil erosion processes under complex cultivation patterns on the same field. Modeling capabilities should be improved and tested with respect to the soil erosion process in strip and patch cropping systems as well as agroforestry systems. Existing agroforestry models must be improved to incorporate erosion processes in fields with high spatial heterogeneity with respect to soil properties, slope inclination, and length, preferably considering three dimensions. Such new developments might also support upscaling of soil erosion processes to larger spatial scales (watershed to basin scale).

\subsection{Summary and Conclusions}

Soil erosion processes strongly differ with spatial and temporal scales and environmental conditions. Thus, a large number of models have been developed that differ in terms of the processes considered, temporal and spatial application scale, capabilities, and limitations. Based on their processing concepts, these models were classified into three categories (1) Empirical models, (2) Conceptual models, (3) Physically based models.

Most of the empirical models use the universal soil loss equation (USLE) and its derivates RUSLE and MUSLE. Though these equations have been developed using data obtained in the United States, these equations are applied worldwide for soil loss estimations. Under variable conditions of spatial soil characteristics and insufficient meteorological 
networks, empirical models are less complex to operate which makes them a potential choice for predicting soil erosion. Hence, the empirical models are more likely to be used with limited availability of input data. Contrary to that, physically based models provide a physical description of the erosion process. These models are comparatively complex and less user-friendly because of their detailed depictions of processes and large data requirements. However, physically based models are more capable when performing event-based simulations. Conceptual models typically have been developed for catchment and larger scales, requiring a general description of the catchment and involved soil erosion processes, without describing the details of their interactions that would require big data sets of temporal and spatially distributed catchment details. However, there is currently no model available to represent soil erosion processes in more complex cropping at the field scale like strip and patch cropping or alley cropping systems. There are some crop models available to simulate alley cropping systems such as APSIM or WaNuLCAS, with soil erosion components. However, these models have not been tested and validated for erosion estimation and its impacts on subsequent crop yield.

The literature review indicated that most of the models developed for large agriculture catchments using equations developed under specific conditions require site-specific calibration before simulation. Models designed for small time steps perform better than continuous scale modeling. Similarly, calibration at a field or smaller scale, where spatial topographic and soil variations on erosion process greatly affect the simulation, is more accurate than that of larger catchment areas. It is worth indicating that some models such as EPIC, PERFECT, GUEST, EPM, TCRP, APSIM, and CREAMS were developed for soil erosion assessment at plot/field scale at daily time steps. Limited workability of these models was found for sediment transport, sediment deposit, and sediment yield. Models such as EPIC, WEPP, EUROSEM, CREAMS, SCUAF, and WaNuLCAS have the capability to account for rainfall interception but further improvements are required to deal with complex cropping systems.

\section{A Way Forward}

Soil erosion modeling at a field scale is now facilitated by very high spatial and temporal resolution remote sensing (RS) data, which allow for frequent estimation of characteristics of crop cover and topsoil characteristics at the field scale. RS data can be used as both model input (e.g., microtopography, within-field variability of oil and plant characteristics) and validation data (e.g. based on LiDAR data [173]). In order to benefit from RS data flexible data assimilation methods have to be developed for physically based models whereas their integration into empirical and conceptual models is relatively straightforward. At larger scales, EU wide surveys of topsoil (LUCAS) and Land Use/Cover Area (CORINE) are carried out every three years. Such harmonized open-access data are currently not fully exploited by soil erosion models and might be used as input data for urgently needed model intercomparisons in order to increase our confidence in predicted erosion rates. Further, with increased concerns on future soil erosion rates under climate change [174] the systematic evaluation of soil erosion models and ensemble soil erosion models [175] using harmonized data sets might be used to support land use policies.

Models such as EPIC, WEPP, EUROSEM, and CREAMS may have the capabilities to model complex cropping systems such as strip cropping and patch cropping, their spatial arrangements, and their impact on soil erosion when integrated with GIS or into flexible modeling frameworks. Integration or coupling of soil erosion components in a modeling framework for dynamic simulation can provide an alternative to conventional erosion modeling at a field scale and may facilitate upscaling to larger scales.

In the context of sustainable agriculture, there has been an increasing interest in the application of novel crop arrangements within a field [176] in recent years. Since these novel field designs, e.g. alley cropping or strip cropping, are assumed to support the delivery of ecosystem services, such as a reduction of soil erosion, there is a need to quantify such effects. A larger number of case studies, based on the combined use of high-resolution 
RS data with soil erosion models, are required in order to highlight the potential of novel field designs to reduce the risk of soil erosion and to support corresponding changes in agricultural policy. An important conclusion of this review is, therefore, the need for future research and development with respect to modeling soil erosion under complex spatial cultivation patterns.

The present study provides a clear description of individual models to sort out which model fits which conditions and problems identified and leads to clear guidelines to select the appropriate model. Future studies need to integrate modeling working components to enhance the strength of models. There should be models developed for soil erosion process in agroforestry systems and existing agroforestry models must be improved to incorporate erosion process and yields. This review emphasizes enhancing the quality of the modeling output and should have additional components to enhance their applicability in most environmental and management conditions.

Author Contributions: Conceptualization, A.R., T.G., M.H.-U.-R., and H.A.; methodology, A.R., T.G., M.H.-U.-R., and H.A.; resource and data curation, A.R., T.G., and H.A., writing-original draft preparation, A.R.; writing-review and editing, T.G., H.A., M.H.-U.-R., and A.R.; visualization, H.A., and T.G.; supervision, T.G.; project administration, T.G.; funding acquisition, T.G., H.A. All authors have read and agreed to the published version of the manuscript.

Funding: This research was funded by the German Federal Ministry of Education and Research (BMBF) through the Digital Agriculture Knowledge and Information System (DAKIS) Project, grant number 031B0729E, and the APC was funded by the Crop Science Group, University of Bonn, Germany.

Institutional Review Board Statement: Not applicable.

Informed Consent Statement: Not applicable.

Data Availability Statement: The datasets generated during and/or analyzed during the current study are available from the corresponding author on reasonable request.

Acknowledgments: We would like to thank all reviewers for their efforts and fruitful feedback on the article.

Conflicts of Interest: The authors declare no conflict of interest.

\section{References}

1. Boardman, J.; Poesen, J. Soil Erosion in Europe: Major Processes, Causes and Consequences. In Soil Erosion in Europe; John Wiley and Sons Ltd.: Hoboken, NJ, USA, 2006; pp. 477-487. ISBN 9780470859209.

2. Phuong, T.T.; Shrestha, R.P.; Chuong, H.V. Simulation of Soil Erosion Risk in the Upstream Area of Bo River Watershed. In Redefining Diversity and Dynamics of Natural Resources Management in Asia; Elsevier Inc.: Amsterdam, The Netherlands, 2017; Volume 3, pp. 87-99. ISBN 9780128104743.

3. Liu, Y.H.; Li, D.H.; Chen, W.; Lin, B.S.; Seeboonruang, U.; Tsai, F. Soil erosion modeling and comparison using slope units and grid cells in Shihmen reservoir watershed in Northern Taiwan. Water 2018, 10, 1387. [CrossRef]

4. Ahamefule, H.; Fatola, F.; Olaniyan, J.; Amana, M.; Eifediyi, E.; Ihem, E.; Nwokocha, C.; Adepoju, A.; Adepoju, I.; Babalola, M. Prediction Models for Water Erosion Risk Management: A Review. J. Appl. Sci. Environ. Manag. 2018, 22, 1389. [CrossRef]

5. Merritt, W.S.; Letcher, R.A.; Jakeman, A.J. A review of erosion and sediment transport models. Environ. Model. Softw. 2003, 18, 761-799. [CrossRef]

6. Swarnkar, S.; Malini, A.; Tripathi, S.; Sinha, R. Assessment of uncertainties in soil erosion and sediment yield estimates at ungauged basins: An application to the Garra River basin, India. Hydrol. Earth Syst. Sci. 2018, 22, 2471-2485. [CrossRef]

7. Favis-Mortlock, D.; Mullan, D. Soil erosion by water under future climate change. In Soil Hydrology, Land use and Agriculture: Measurement and Modelling; CABI Publishing: Wallingford, UK, 2011; pp. 384-414. ISBN 9781845937973.

8. Parsons, A.J.; Wainwright, J.; Mark Powell, D.; Kaduk, J.; Brazier, R.E. A conceptual model for determining soil erosion by water. Earth Surf. Process. Landf. 2004, 29, 1293-1302. [CrossRef]

9. Ranzi, R.; Bochicchio, M.; Bacchi, B. Effects on floods of recent afforestation and urbanisation in the Mella River (Italian Alps). Hydrol. Earth Syst. Sci. 2002, 6, 239-253. [CrossRef]

10. Doe, W.; Jones, D.; Warren, S. The soil erosion model guide for military land mangers: Analysis of erosion models for natural and cultural resources applications. US Army Eng. Waterw. Exp. Stn. Tech. Rept. ITL 1999, 99-XX, 122p.

11. Renschler, C.S.; Harbor, J. Soil erosion assessment tools from point to regional scales-The role of geomorphologists in land management research and implementation. Geomorphology 2002, 47, 189-209. [CrossRef] 
12. Haregeweyn, N.; Yohannes, F. Testing and evaluation of the agricultural non-point source pollution model (AGNPS) on Augucho catchment, western Hararghe, Ethiopia. Agric. Ecosyst. Environ. 2003, 99, 201-212. [CrossRef]

13. El Jazouli, A.; Barakat, A.; Ghafiri, A.; El Moutaki, S.; Ettaqy, A.; Khellouk, R. Soil erosion modeled with USLE, GIS, and remote sensing: A case study of Ikkour watershed in Middle Atlas (Morocco). Geosci. Lett. 2017, 4, 25. [CrossRef]

14. Patil, R.J.; Sharma, S.K.; Tignath, S. Remote Sensing and GIS based soil erosion assessment from an agricultural watershed. Arab. J. Geosci. 2015, 8, 6967-6984. [CrossRef]

15. Favis-Mortlock, D.; Boardman, J.; MacMillan, V. Chapter 16 The Limits of Erosion Modeling. Landsc. Eros. Evol. Model. 2001, 477-516.

16. Favis-Mortlock, D.T.; Boardman, J.; Parsons, A.J.; Lascelles, B. Emergence and erosion: A model for rill initiation and development. Hydrol. Process. 2000, 14, 2173-2205. [CrossRef]

17. Kirkby, M.J.; Abrahart, R.; McMahon, M.D.; Shao, J.; Thornes, J.B. MEDALUS soil erosion models for global change. Geomorphology 1998, 24, 35-49. [CrossRef]

18. Monjezi, A.; Masjedi, A.; Heidarnejad, M.; Pourmohammadi, M.H. Effects of slot size in the groin body on the riprap stability in a river bend. Fresenius Environ. Bull. 2017, 26, 7034-7044.

19. Alewell, C.; Borrelli, P.; Meusburger, K.; Panagos, P. Using the USLE: Chances, challenges and limitations of soil erosion modelling. Int. Soil Water Conserv. Res. 2019, 7, 203-225. [CrossRef]

20. Eisazadeh, L.; Sokouti, R.; Homaee, M.; Pazira, E. Comparison of empirical models to estimate soil erosion and sediment yield in micro catchments. Eurasian J. Soil Sci. 2012, 1, 28-33. [CrossRef]

21. Santos, C.A.G.; Watanabe, M.; Suzuki, K. A Conceptual Soil Erosion Model. Proc. Hydraul. Eng. 1998, 42, 1033-1038. [CrossRef]

22. Probert, M.E.; Dimes, J.P.; Keating, B.A.; Dalal, R.C.; Strong, W.M. APSIM's water and nitrogen modules and simulation of the dynamics of water and nitrogen in fallow systems. Agric. Syst. 1998, 56, 1-28. [CrossRef]

23. Wischmeier, W.H.; Smith, D.D. Predicting Rainfall-Erosion Losses from Cropland East of the Rocky Mountains: Guide for Selection of Practices for Soil and Water Conservation by Wischmeier, W.H., Dwight David Smith-Books on Google Play; U.S. Department of Agriculture: Washington, DC, USA, 1949.

24. Albaladejo Montoro, J.; Stocking, M. Comparative evaluation of two models in predicting storm soil loss from erosion plots in semi-arid Spain. Catena 1989, 16, 227-236. [CrossRef]

25. Williams, J.R.; Berndt, H.D. Sediment Yield Prediction Based on Watershed Hydrology. Trans. ASAE 1977, $20,1100-1104$. [CrossRef]

26. Chandramohan, T.; Venkatesh, B.; Balchand, A.N. Evaluation of Three Soil Erosion Models for Small Watersheds. Aquat. Procedia 2015, 4, 1227-1234. [CrossRef]

27. Renard, K.G.; Foster, G.R.; Weesies, G.A.; Porter, J.P. RUSLE: Revised universal soil loss equation. J. Soil Water Conserv. 1991, 46, 30-33.

28. Le Roux, J.J.; Morgenthal, T.L.; Malherbe, J.; Pretorius, D.J.; Sumner, P.D. Water erosion prediction at a national scale for South Africa. Water SA 2008, 3, 305-314. [CrossRef]

29. Charles, R.; Meyer, L.E.; Wagner, D.C.; Yoder, D.C. Flanagan The Modular Soil Erosion System (MOSES). Soil Eros. Res. 21st Century 2001, 361. [CrossRef]

30. Ferro, V.; Porto, P. Sediment Delivery Distributed (SEDD) Model. J. Hydrol. Eng. 2000, 5, 411-422. [CrossRef]

31. Dragičević, N.; Karleuša, B.; Ožanić, N. Erosion potential method (Gavrilović Method) sensitivity analysis. Soil Water Res. 2017, 12, 51-59. [CrossRef]

32. Ahmadi, M.; Minaei, M.; Ebrahimi, O.; Nikseresht, M. Evaluation of WEPP and EPM for improved predictions of soil erosion in mountainous watersheds: A case study of Kangir River basin, Iran. Model. Earth Syst. Environ. 2020, 6, 2303-2315. [CrossRef]

33. Takken, I.; Jetten, V.; Govers, G.; Nachtergaele, J.; Steegen, A. The effect of tillage-induced roughness on runoff and erosion patterns. Geomorphology 2001, 37, 1-14. [CrossRef]

34. Thaxton, C.S.; Mitasova, H.; Mitas, L.; McLaughlin, R. Simulations of distributed watershed erosion, deposition, and terrain evolution using a path sampling Monte Carlo method. In Proceedings of the ASAE Annual International Meeting 2004, Ottawa, ON, Canada, 1-4 August 2004; American Society of Agricultural and Biological Engineers: St. Joseph, MI, USA; pp. $2069-2082$.

35. Elwell, H.A. Modelling soil losses in Southern Africa. J. Agric. Eng. Res. 1978, 23, 117-127. [CrossRef]

36. PSIAC. Report of the Water Management Subcommittee on Factors Affecting Sediment Yield in the Pacific Southwest Area and Selectionand Evaluation of Measures for Reduction of Erosion and Sediment Yield; Pacific Southwest Inter-Agency Committee: Portland, Oregon, 1968.

37. De Vente, J.; Poesen, J.; Verstraeten, G.; Govers, G.; Vanmaercke, M.; Van Rompaey, A.; Arabkhedri, M.; Boix-Fayos, C. Predicting soil erosion and sediment yield at regional scales: Where do we stand? Earth Sci. Rev. 2013, 127, 16-29. [CrossRef]

38. Kiyoshi, H. Evaluation of vegetation change in the Asio copper mine using remote sensing and its application to forest conservation works. In Proceedings of the Workshop on the Application of Remote Sensing Technology to Natural Disaster Reduction; Japan-United States Science and Technology Agreement (JUST): Tsukuba, Japan, 1993; Volume 5, pp. 147-149.

39. Van Noordwijk, M.; Lusiana, B. WaNulCAS, a model of water, nutrient and light capture in agroforestry systems. Agrofor. Syst. 1998, 43, 217-242. [CrossRef]

40. Onsamrarn, W.; Chittamart, N.; Tawornpruek, S. Performances of the WEPP and WaNuLCAS models on soil erosion simulation in a tropical hillslope, Thailand. PLoS ONE 2020, 15. [CrossRef] [PubMed] 
41. Young, A. Agroforestry for Soil Conservation; CAB International: Wallingford, UK, 1989.

42. Beck, M.B. Water quality modeling: A review of the analysis of uncertainty. Water Resour. Res. 1987, 23, 1393-1442. [CrossRef]

43. Chmelová, R.; Šarapatka, B. Soil erosion by water: Contemporary research methods and their use. Geographica 2002, 37, 23-30.

44. Sorooshian, S. Parameter estimation, model identification, and model validation: Conceptual-type models. Recent Adv. Model. Hydrol. Syst. 1991, 443-467. [CrossRef]

45. Jakeman, A.J.; Hornberger, G.M. How much complexity is warranted in a rainfall-runoff model? Water Resour. Res. 1993, 29, 2637-2649. [CrossRef]

46. Abbott, M.B.; Bathurst, J.C.; Cunge, J.A.; O'Connell, P.E.; Rasmussen, J. An introduction to the European Hydrological SystemSysteme Hydrologique Europeen, "SHE", 1: History and philosophy of a physically-based, distributed modelling system. J. Hydrol. 1986, 87, 45-59. [CrossRef]

47. Kleissen, F.M. Uncertainty and Identifiability in Conceptual Models of Surface Water Acidification. Master's Thesis, University of London, London, UK, 1990.

48. Pechlivanidis, I.G.; Jackson, B.M.; Mcintyre, N.R.; Wheater, H.S. Catchment scale hydrological modelling: A review of model types, calibration approaches and uncertainty analysis methods in the context of recent developments in technology and applications. Glob. Int. J. 2011, 13, 193-214.

49. Onof, C.; Wheater, H.S. Modelling of British rainfall using a random parameter Bartlett-Lewis Rectangular Pulse Model. J. Hydrol. 1993, 149, 67-95. [CrossRef]

50. McCown, R.L.; Hammer, G.L.; Hargreaves, J.N.G.; Holzworth, D.P.; Freebairn, D.M. APSIM: A novel software system for model development, model testing and simulation in agricultural systems research. Agric. Syst. 1996, 50, 255-271. [CrossRef]

51. Basche, A.D.; Archontoulis, S.V.; Kaspar, T.C.; Jaynes, D.B.; Parkin, T.B.; Miguez, F.E. Simulating long-term impacts of cover crops and climate change on crop production and environmental outcomes in the Midwestern United States. Agric. Ecosyst. Environ. 2016. [CrossRef]

52. Favis-Mortlock, D.T. An evolutionary approach to the simulation of rill initiation and development. Proc. first Int. Conf. Geocomputation 1996, 1, 248-281.

53. Arnold, J.G.; Srinivasan, R.; Muttiah, R.S.; Williams, J.R. Large area hydrologic modeling and assessment part I: Model development. J. Am. Water Resour. Assoc. 1998, 34, 73-89. [CrossRef]

54. Aga, A.O.; Melesse, A.M.; Chane, B. An alternative empirical model to estimate watershed sediment yield based on hydrology and geomorphology of the basin in data-scarce rift VALLEY lake regions, Ethiopia. Geoscience 2020, 10, 31. [CrossRef]

55. Krysanova, V.; Müller-Wohlfeil, D.I.; Becker, A. Development and test of a spatially distributed hydrological/water quality model for mesoscale watersheds. Ecol. Modell. 1998, 106, 261-289. [CrossRef]

56. Krysanova, V.; Hattermann, F.; Wechsung, F. Implications of complexity and uncertainty for integrated modelling and impact assessment in river basins. Environ. Model. Softw. 2007, 22, 701-709. [CrossRef]

57. Simons, M.; Podger, G.; Cooke, R. IQQM-A hydrologic modelling tool for water resource and salinity management. Environ. Softw. 1996, 11, 185-192. [CrossRef]

58. Murray, A.B.; Paola, C. A cellular model of braided rivers. Nature 1994, 371, 54-57. [CrossRef]

59. Coulthard, T.J.; Kirkby, M.J.; Macklin, M.G. Modelling geomorphic response to environmental change in an upland catchment. Hydrol. Process. 2000, 14, 2031-2045. [CrossRef]

60. Kirkby, M.J. Hydrograph Modelling Strategies. In Processes in Physical and Human Geography; University of Leeds: Ledds, UK, 1975; pp. 69-90.

61. Kirkby, M.J. TOPMODEL: A PERSONAL VIEW. Hydrol. Process. 1997, 11, 1087-1097. [CrossRef]

62. Beven, K.J.; Kirkby, M.J.; Schofield, N.; Tagg, A.F. Testing a physically-based flood forecasting model (TOPMODEL) for three U.K. catchments. J. Hydrol. 1984, 69, 119-143. [CrossRef]

63. Luo, W.; Duffin, K.L.; Peronja, E.; Stravers, J.A.; Henry, G.M. A web-based interactive landform simulation model (WILSIM). Comput. Geosci. 2004, 30, 215-220. [CrossRef]

64. Luo, W.; Peronja, E.; Duffin, K.; Stravers, J.A. Incorporating nonlinear rules in a web-based interactive landform simulation model (WILSIM). Comput. Geosci. 2006, 32, 1512-1518. [CrossRef]

65. Williams, J.R.; Nicks, A.D.; Arnold, J.G. Simulator for Water Resources in Rural Basins. J. Hydraul. Eng. 1985, 111, 970-986. [CrossRef]

66. Sivapalan, M.; Viney, N.R.; Ruprecht, J.K. Water and salt balance modelling to predict the effects of land-use changes in forested catchments. 2. Coupled model of water and salt balances. Hydrol. Process. 1996, 10, 413-428. [CrossRef]

67. Sivapalan, M.; Viney, N.R.; Zammit, C.; Singh, V.P.; Frevert, D.K. LASCAM: Large scale catchment model. Math. Model. Large Watershed Hydrol. 2002, 579-648.

68. Young, R.A.; Onstad, C.A.; Bosch, D.D.; Anderson, W.P. AGNPS: A nonpoint-source pollution model for evaluating agricultural watersheds. J. Soil Water Conserv. 1989, 44, 168-173.

69. Schulze, R.E. Hydrology and Agrohydrology-A Text to Accompany the ACRU 3.00 Agrohydrological Systesm; Dept. of Agricultural Engineering University of Natal.: Pietermaritzburg, South Africa, 1995.

70. Aduah, M.S.; Jewitt, G.P.W.; Warburton Toucher, M.L. Assessing suitability of the ACRU hydrological model in a rainforest catchment in Ghana, West Africa. Water Sci. 2017, 31, 198-214. [CrossRef] 
71. King, C.; Lecomte, V.; Le Bissonnais, Y.; Baghdadi, N.; Souchère, V.; Cerdan, O. Remote-sensing data as an alternative input for the "STREAM" runoff model. Catena 2005, 62, 125-135. [CrossRef]

72. Karydas, C.G.; Panagos, P.; Gitas, I.Z. A classification of water erosion models according to their geospatial characteristics. Int. J. Digit. Earth 2014, 7, 229-250. [CrossRef]

73. Grunwald, S.; Haverkamp, S.; Bach, M.; Frede, H.G. Uberprufung von MEKA-Massnahmen zum Erosions-und Gewasserschutz durch das Modell AGNPSm. Z. Kult Landentwickl. 1997, 38, 260-265.

74. Mohamadi, M.A.; Kavian, A. Effects of rainfall patterns on runoff and soil erosion in field plots. Int. Soil Water Conserv. Res. 2015, 3, 273-281. [CrossRef]

75. Kandel, D.D.; Western, A.W.; Grayson, R.B.; Turral, H.N. Process parameterization and temporal scaling in surface runoff and erosion modelling. Hydrol. Process. 2004, 18, 1423-1446. [CrossRef]

76. Lighthill, M.J.; Whitham, G.B. On kinematic waves I. Flood movement in long rivers. In Proceedings of the Royal Society of London. Series a Mathematical and Physical Sciences; The Royal Society: London, UK, 1955; Volume 229, pp. 281-316.

77. Dunin, F.X. Use of physical process models. In Proceedings of the Prediction in Catchment Hydrology; National Symposium on Hydrology, Canberra, Australia, 25-27 November 1975; Australian Academy of Science: Canberra, Australia, 1975 ; pp. $277-291$.

78. Beven, K.J.; Kirkby, M.J. A physically based, variable contributing area model of basin hydrology. Hydrol. Sci. Bull. 1979, 24, 43-69. [CrossRef]

79. Seyfried, M.S.; Wilcox, B.P. Scale and the Nature of Spatial Variability: Field Examples Having Implications for Hydrologic Modeling. Water Resour. Res. 1995, 31, 173-184. [CrossRef]

80. Blöschl, G.; Sivapalan, M. Scale issues in hydrological modelling: A review. Hydrol. Process. 1995, 9, 251-290. [CrossRef]

81. Beasley, D.B.; Huggins, L.F.; Monke, E.J. ANSWERS: A model for watershed planning. Trans. Am. Soc. Agric. Eng. 1980, 23, 938-944. [CrossRef]

82. Bouraoui, F.; Dillaha, T.A. ANSWERS-2000: Runoff and Sediment Transport Model. J. Environ. Eng. 1996, 122, 493-502. [CrossRef]

83. Williams, J.R.; Jones, C.A.; Kiniry, J.R.; Spanel, D.A. The EPIC Crop Growth Model. Trans. ASAE 1989, 32, 0497-0511. [CrossRef]

84. Pumijumnong, N.; Arunrat, N. Reliability and Evaluation of the Potential of the i_EPIC Model to Estimate Rice Yields in Thailand. Agric. Sci. Res. J. 2012, 2, 614-622.

85. Watson, D.A.; Laflen, J.M.; Franti, T.G. Estimating Ephemeral Gully Erosion. In Proceedings of the Paper-American Society of Agricultural Engineers, Boulder, Colorado, 11-14 June 1986; American Society of Agricultural Engineers: St. Joseph, MI, USA, 1986.

86. Woodward, D.E. Method to predict cropland ephemeral gully erosion. Catena 1999, 37, 393-399. [CrossRef]

87. Borah, D.K. Runoff Simulation Model for Small Watersheds. Trans. ASAE 1989, 32, 0881-0886. [CrossRef]

88. Knisel, W.G. Creams a Field-Scale Model for Chemicals, Runoff, and Erosion from Agricultural Management Systems; Model Docu.; Dept. of Agriculture, Science and Education Administration: Corvallis, OR, USA, 1982; Volume 1, ISBN 0876647158.

89. Schmidt, J. A mathematical model to simulate rainfall erosion. Catena Suppl. 1991, 19, 101-109.

90. Schmidt, J.; Werner, M.V.; Michael, A. Application of the EROSION 3D model to the CATSOP watershed, the Netherlands. Catena 1999, 37, 449-456. [CrossRef]

91. Morgan, R.P.C.; Quinton, J.N.; Smith, R.E.; Govers, G.; Prosen, J.W.A.; Auerswald, K.; Chisci, G.; Torri, D.; Styczen, M.E.; Folley, A.J.V. The European Soil Erosion Model (EUROSEM): Documentation and User Guide; Cranfield University: Oxfordshire, UK, 1998.

92. Khaleghpanah, N.; Shorafa, M.; Asadi, H.; Gorji, M.; Davari, M. Modeling soil loss at plot scale with EUROSEM and RUSLE2 at stony soils of Khamesan watershed, Iran. Catena 2016, 147, 773-788. [CrossRef]

93. Hairsine, P.B.; Rose, C.W. Rainfall Detachment and Deposition: Sediment Transport in the Absence of Flow-Driven Processes. Soil Sci. Soc. Am. J. 1991, 55, 320. [CrossRef]

94. Misra, R.K.; Rose, C.W. Application and sensitivity analysis of process-based erosion model GUEST. Eur. J. Soil Sci. 1996, 47, 593-604. [CrossRef]

95. Singh, V.P.; Frevert, D.K. Watershed Models. Available online: https://www.worldcat.org/title/watershed-models/oclc/770075 54 (accessed on 2 December 2020).

96. Leonard, R.A.; Knisel, W.G.; Still, D.A. Gleams: Groundwater loading effects of agricultural management systems. Trans. ASAE 1987, 30, 1403-1418. [CrossRef]

97. Garnier, M.; Lo Porto, A.; Marini, R.; Leone, A. Integrated use of GLEAMS and GIS to prevent groundwater pollution caused by agricultural disposal of animal waste. Environ. Manage. 1998, 22, 747-756. [CrossRef] [PubMed]

98. Woolhiser, D.A.; Smith, R.E.; Goodrich, D.C. A Kinematic Runoff and Erosion Model; Documentation and User: Washington, DC, USA, 1990.

99. Tajbakhsh, S.M.; Memarian, H.; Sobhani, M.; Aghakhani Afshar, A.H. Kinematic runoffand erosion model efficiency assessment for hydrological simulation of semi-arid watersheds. Glob. J. Environ. Sci. Manag. 2018, 4, 127-140. [CrossRef]

100. Viney, N.R.; Sivapalan, M. A conceptual model of sediment transport: Application to the Avon River Basin in Western Australia. Hydrol. Process. 1999, 13, 727-743. [CrossRef]

101. Seixas, J.; Nuno Vieira, G.; Pedro Nunes, J. Mefidis: A Physically Based, Spatially Distributed Runoff and Erosion Model for Extreme Rainfall Events. Environ. Sci. 2005, 291-313. [CrossRef]

102. Kirkby, M. Modelling Across Scales: The Medalus Family of Models. In Modelling Soil Erosion by Water; Springer: Berlin/Heidelberg, Germany, 1998; pp. 161-173. 
103. Lamqadem, A.A.; Pradhan, B.; Saber, H.; Rahimi, A. Desertification sensitivity analysis using medalus model and gis: A case study of the oases of middle draa valley, morocco. Sensors 2018, 18, 2230. [CrossRef] [PubMed]

104. Littleboy, M.; Silburn, D.M.; Freebairn, D.M.; Woodruff, D.R.; Hammer, G.L. PERFECT: A computer simulation model of Productivity, Erosion, Runoff Functions to Evaluate Conservation Techniques. Queensl. Dep. Prim. Ind. Brisb. QLD Bull. QB 1989, 9005, 119.

105. Littleboy, M.; Silburn, D.M.; Freebaim, D.M.; Woodruf, D.R.; Hammer, G.L.; Leslie, J.K. Impact of soil erosion on production in cropping systems. I. development and validation of a simulation model. Aust. J. Soil Res. 1992, 30, 757-774. [CrossRef]

106. Schramm, M. Ein Erosionsmodell mit Räumlich und Zeitlich Veränderlicher Rillenmorphologie; Universität Fridericiana zu Karlsruhe: Karlsruhe, Germany, 1994.

107. Kirkby, M.J.; Jones, R.; Irvine, B.; Gobin, A.; Govers, G.; Cerdan, O.; Van Rompaey, A.J.J.; Le Bissonnais, Y.; Daroussin, J.; King, D.; et al. Pan-European Soil Erosion Risk Assessment: The PESERA Map, Version 1 October 2003. Explanation of Special Publication Ispra 2004 No.73 (S.P.I.04.73); European Commission: Luxembourg, 2004; Volume 16.

108. Laflen, J.M.; Lane, L.J.; Foster, G.R. WEPP: A new generation of erosion prediction technology. J. Soil Water Conserv. 1991, 46, 34-38.

109. Brooks, E.S.; Dobre, M.; Elliot, W.J.; Wu, J.Q.; Boll, J. Watershed-scale evaluation of the Water Erosion Prediction Project (WEPP) model in the Lake Tahoe basin. J. Hydrol. 2016, 533, 389-402. [CrossRef]

110. Lopes, L.V. A Numerical Model of Watershed Erosion and Sediment Yield Item Type Dissertation-Reproduction (Electronic); University of Arizona: Tucson, AZ, USA, 1987.

111. Van Oost, K.; Govers, G.; Desmet, P. Evaluating the effects of changes in landscape structure on soil erosion by water and tillage. Lanscape Ecol. 2000, 15, 577-589. [CrossRef]

112. Panagos, P.; Katsoyiannis, A. Soil erosion modelling: The new challenges as the result of policy developments in Europe. Environ. Res. 2019, 172, 470-474. [CrossRef] [PubMed]

113. De Jong, S.M.; Paracchini, M.L.; Bertolo, F.; Folving, S.; Megier, J.; De Roo, A.P.J. Regional assessment of soil erosion using the distributed model SEMMED and remotely sensed data. Catena 1999, 37, 291-308. [CrossRef]

114. Fernandes, J.; Bateira, C.; Soares, L.; Faria, A.; Oliveira, A.; Hermenegildo, C.; Moura, R.; Gonçalves, J. SIMWE model application on susceptibility analysis to bank gully erosion in Alto Douro Wine Region agricultural terraces. Catena 2017, 153, 39-49. [CrossRef]

115. Nearing, M.A.; Wei, H.; Stone, J.J.; Pierson, F.B.; Spaeth, K.E.; Weltz, M.A.; Flanagan, D.C.; Hernandez, M. A Rangeland Hydrology and Erosion Model. Trans. ASABE 2011, 54, 901-908. [CrossRef]

116. Hernandez, M.; Nearing, M.A.; Al-Hamdan, O.Z.; Pierson, F.B.; Armendariz, G.; Weltz, M.A.; Spaeth, K.E.; Williams, C.J.; Nouwakpo, S.K.; Goodrich, D.C.; et al. The Rangeland Hydrology and Erosion Model: A Dynamic Approach for Predicting Soil Loss on Rangelands. Water Resour. Res. 2017, 53, 9368-9391. [CrossRef]

117. Vertessy, R.A.; Wilson, C.J. Predicting erosion hazard areas using digital terrain analysis. In Proceedings of the Research Needs and Applications to Reduce Erosion and Sedimentation in Tropical Steeplands; IAHS-AISH Publ. No.192, Suva, Fiji, 11-15 June 1990; pp. 298-308.

118. Maftei, C.; Buta, C.; Chevallier, P. Application of the TOPOG model on a flash-flood-prone hill catchment in Romania. J. Environ. Prot. Ecol. 2019, 1, 123-134.

119. Lu, D.; Li, G.; Valladares, G.S.; Batistella, M. Mapping soil erosion risk in Rondônia, Brazilian Amazonia: Using RUSLE, remote sensing and GIS. Land Degrad. Dev. 2004, 15, 499-512. [CrossRef]

120. Pandey, A.; Chowdary, V.M.; Mal, B.C. Identification of critical erosion prone areas in the small agricultural watershed using USLE, GIS and remote sensing. Water Resour. Manag. 2007, 21, 729-746. [CrossRef]

121. DeVantier, B.A.; Feldman, A.D. Review of GIS Applications in Hydrologic Modeling. J. Water Resour. Plan. Manag. 1993, 119, 246-261. [CrossRef]

122. Jenson, S.K.; Domingue, J.O. Extracting Topographic Structure from Digital Elevation Data for Geographic Information System Analysis. Photogramm. Eng. Remote Sens. 1998, 54, 1593-1600.

123. Walker, J.P.; Willgoose, G.R. On the effect of digital elevation model accuracy on hydrology and geomorphology. Water Resour. Res. 1999, 35, 2259-2268. [CrossRef]

124. Coveney, S.; Fotheringham, A.S. The impact of DEM data source on prediction of flooding and erosion risk due to sea-level rise. Int. J. Geogr. Inf. Sci. 2011, 25, 1191-1211. [CrossRef]

125. Karamage, F.; Zhang, C.; Liu, T.; Maganda, A.; Isabwe, A. Soil erosion risk assessment in Uganda. Forests 2017, 8, 52. [CrossRef]

126. Morgan, R.P.C.; Quinton, J.N.; Smith, R.E.; Govers, G.; Poesen, J.W.A.; Auerswald, K.; Chisci, G.; Torri, D.; Styczen, M.E. The European soil erosion model (EUROSEM): A dynamic approach for predicting sediment transport from fields and small catchments. Earth Surf. Process. Landf. 1998, 23, 527-544. [CrossRef]

127. Karydas, C.G.; Panagos, P. Modelling monthly soil losses and sediment yields in Cyprus. Int. J. Digit. Earth 2016, 9, 766-787. [CrossRef]

128. Tavares, A.S.; Spalevic, V.; Avanzi, J.C.; Nogueira, D.A.; Silva, M.L.N.; Mincato, R.L. Modeling of water erosion by the erosion potential method in a pilot subbasin in southern Minas Gerais. Semin. Agrar. 2019, 40, 555-572. [CrossRef]

129. Van Oost, K.; Beuselinck, L.; Hairsine, P.B.; Govers, G. Spatial evaluation of a multi-class sediment transport and deposition model. Earth Surf. Process. Landf. 2004, 29, 1027-1044. [CrossRef] 
130. Heydarnejad, S.; Fordoei, A.R.; Mousavi, S.H.; Mirzaei, R. Estimation of soil erosion using SLEMSA model and OWA approach in Lorestan Province ( Iran ). Environ. Resour. Res. 2020, 8, 11-24.

131. Dilla, A.M.; Smethurst, P.J.; Huth, N.I.; Barry, K.M. Plot-scale agroforestry modeling explores tree pruning and fertilizer interactions for maize production in a Faidherbia parkland. Forests 2020, 11, 1175. [CrossRef]

132. Stolpe, N.B. A comparison of the RUSLE, EPIC and WEPP erosion models as calibrated to climate and soil of south-central Chile. Acta Agric. Scand. Sect. B Soil Plant Sci. 2005, 55, 2-8. [CrossRef]

133. Rekolainen, S.; Posch, M. Adapting the CREAMS model for Finnish conditions. Nord. Hydrol. 1993, 24, 309-322. [CrossRef]

134. Mahmoodabadi, M.; Ghadiri, H.; Rose, C.; Yu, B.; Rafahi, H.; Rouhipour, H. Evaluation of GUEST and WEPP with a new approach for the determination of sediment transport capacity. J. Hydrol. 2014, 513, 413-421. [CrossRef]

135. Palosuo, T.; Christian Kersebaum, K.; Angulo, C.; Hlavinka, P.; Moriondo, M.; Olesen, J.E.; Patil, R.H.; Oise Ruget, F.; Rumbaur, C.; Takáč, J.; et al. Simulation of winter wheat yield and its variability in different climates of Europe: A comparison of eight crop growth models. Eur. J. Agron. 2011, 35, 103-114. [CrossRef]

136. Kouhpeima, A.; Ali, S.; Hashemi, A.; Feiznia, S. A study on the efficiency of Erosion Potential Model (EPM ) using reservoir sediments. Int. J. Environ. Pollut. 2011, 38, 4135-4139.

137. Ali, S.S.; Al-Umary, F.A.; Salar, S.G.; Al-Ansari, N.; Knutsson, S. GIS Based Soil Erosion Estimation Using EPM Method, Garmiyan Area, Kurdistan Region, Iraq. J. Civ. Eng. Archit. 2016, 10, 291-308. [CrossRef]

138. Pandey, S.; Bhandari, H.; Ding, S.; Prapertchob, P.; Sharan, R.; Naik, D.; Taunk, S.K.; Sastri, A. Coping with drought in rice farming in Asia: Insights from a cross-country comparative study. Agric. Econ. 2007, 37, 213-224. [CrossRef]

139. Da Silva, R.M.; Santos, C.A.G.; e Silva, L.P. Evaluation of soil loss in Guaraíra basin by GIS and remote sensing based model. J. Urban Environ. Eng. 2007, 1, 44-52. [CrossRef]

140. Souchere, V.; King, D.; Daroussin, J.; Papy, F.; Capillon, A. Effects of tillage on runoff directions: Consequences on runoff contributing area within agricultural catchments. J. Hydrol. 1998, 206, 256-267. [CrossRef]

141. Souchere, V. Mode 'lisation spatiale du ruissellement a' des fins d'ame'nagement contre l'e 'rosion de thalweg. In Application A'des Petits Bassins Versants en Pays de Caux (Haute-Norman-Die); Institut National Agronomique Paris-Grignon: Paris, France, 1995.

142. Wesseling, C.G.; Karssenberg, D.J.; Burrough, P.A.; Van Deursen, W.P.A. Integrating dynamic environmental models in GIS: The development of a dynamic modelling language. Trans. GIS 1996, 1, 40-48. [CrossRef]

143. Elwell, H.A.; Stocking, M.A. Developing a simple yet practical method of soil-loss estimation. Trop. Agric. 1982.

144. Kinama, J.M.; Stocking, M.; Maingi, P.M. SLEMSA Model Application for Land Use Management in Semi-Arid Kenya. In Proceedings of the Soil Science Society of East Africa Embu, Kenya, East Africa, 26-30 November 2007.

145. Breetzke, D.G.; Koomen, E.S.; Critchley, W.R. GIS-Assisted Modelling of Soil Erosion in a South African Catchment: Evaluating the USLE and SLEMSA Approach. In Water Resources Planning, Development and Management; InTech: London, UK, 2013.

146. Hudson, C.A. A Regional Application of the SLEMSA in the Cathedral Peak Area of the Drakensberg: An Analysis of the Applicability of the Soil Loss Estimation Model for Southern Africa. In Small Mountain Catchments, Soil Conse; University of Cape Town: Cape Town, South Africa, 1987.

147. Masere, T.P.; Worth, S. Applicability of APSIM in decision-making by small-scale resource-constrained farmers: A case of lower Gweru communal area, Zimbabwe. J. Int. Agric. Ext. Educ. 2015, 22, 20-34. [CrossRef]

148. Teixeira, E.I.; de Ruiter, J.; Ausseil, A.G.; Daigneault, A.; Johnstone, P.; Holmes, A.; Tait, A.; Ewert, F. Adapting crop rotations to climate change in regional impact modelling assessments. Sci. Total Environ. 2018, 616-617, 785-795. [CrossRef]

149. Freebairn, D.M.; Wockner, G.H. A study of soil erosion on vertisols of the eastern darling downs, queensland. I effects of surface conditions on soil movement within contour bay catchments. Aust. J. Soil Res. 1986, 24, 135-158. [CrossRef]

150. Horton, R.E. Erosional development of streams and their drainage basins; Hydrophysical approach to quantitative morphology. Bull. Geol. Soc. Am. 1945, 56, 275-370. [CrossRef]

151. Favis-Mortlock, D.; Guerra, T.; Boardman, J. A self-organizing dynamic systems approach to hillslope rill initiation and growth: Model development and validation. In Proceedings of the Modelling Soil Erosion, Sediment Transport and Closely Related Hydrological Processes, Vienna, Austria, 13-17 July 1998; IAHS Publ.: Wallingford, UK; pp. 53-61.

152. Zobeck, T.M.; Onstad, C.A. Tillage and rainfall effects on random roughness: A review. Soil Tillage Res. 1987, 9, 1-20. [CrossRef]

153. Meyer, L.D.; Wischmeier, W.H. Mathematical Simulation of the Process of Soil Erosion by Water. Am. Soc. Agric. Engrs. Trans. 1969, 2, 68-732.

154. Parsons, R.L.; Pease, J.W.; Martens, D.C. Simulating corn yields over 16 years on three soils under inorganic fertilizer and hog manure fertility regimes1. Commun. Soil Sci. Plant Anal. 1995, 26, 1133-1150. [CrossRef]

155. Parton, W.J.; Ojima, D.S.; Cole, C.V.; Schimel, D.S. A General Model for Soil Organic Matter Dynamics: Sensitivity to Litter Chemistry, Texture and Management. In Quantitative Modeling of Soil Forming Processes; Proc. Symposium: Minneapolis, MN, USA, 2015; pp. 147-167.

156. Potter, K.N.; Williams, J.R. Predicting Daily Mean Soil Temperatures in the EPIC Simulation Model. Agron. J. 1994, 86, $1006-1011$. [CrossRef]

157. Cabelguenne, M.; Debaeke, P.; Puech, J.; Bosc, N. Real time irrigation management using the EPIC-PHASE model and weather forecasts. Agric. Water Manag. 1997, 32, 227-238. [CrossRef]

158. Cabelguenne, M.; Jones, C.A.; Williams, J.R. Strategies for limited irrigations of maize in southwestern France-A modeling approach. Trans. Am. Soc. Agric. Eng. 1995, 38, 507-511. [CrossRef] 
159. Roloff, G.; De Jong, R.; Nolin, M.C. Crop yield, soil temperature and sensitivity of EPIC under central-eastern Canadian conditions. Can. J. Soil Sci. 1998, 78, 431-439. [CrossRef]

160. Lee, J.J.; Phillips, D.L.; Liu, R. The effect of trends in tillage practices on erosion and carbon content of soils in the US corn belt. Water Air Soil Pollut. 1993, 70, 389-401. [CrossRef]

161. Apezteguía, H.P.; Izaurralde, R.C.; Sereno, R. Simulation study of soil organic matter dynamics as affected by land use and agricultural practices in semiarid Córdoba, Argentina. Soil Tillage Res. 2009, 102, 101-108. [CrossRef]

162. Smith, S.J.; Williams, J.R.; Hawkins, R.H. Prediction of Sediment Yield from Southern Plains Grasslands with the Universal Soil Loss Equation. J. Range Manag. 1985, 38, 20. [CrossRef]

163. Williams, J.R.; Renard, K.G.; Dyke, P.T. EPIC: A new method for assessing erosion's effect on soil productivity. J. Soil Water Conserv. 1983, 38, 381-383.

164. Nicks, A.D.; Harp, J.F. Stochastic generation of temperature and solar radiation data. J. Hydrol. 1980, 48, 1-17. [CrossRef]

165. Williams, J.R.; Jones, C.A.; Dyke, P.T. A Modeling Approach to Determining the Relationship Between Erosion and Soil Productivity. Trans. ASAE 1984, 27, 0129-0144. [CrossRef]

166. Williams, J.R. The erosion-productivity impact calculator (EPIC) model: A case history. Philos. Trans. R. Soc. London. Ser. B Biol. Sci. 1990, 329, 421-428. [CrossRef]

167. Knisel, W.G.; Nicks, A.D. CREAMS: A Field Scale Model for Chemicals, Runoff, and Errosion from Agriculture Management System, 26th ed.; Dept. of Agriculture, Science and Education Administration: Corvallis, OR, USA, 1981; Volume 1.

168. Mockus, V. Section 4, Deign Hydrographs. In National Engineering Handbook; Mckeever, V., Owen, W., Rallison, R., Eds.; U.S. Dept. of Agriculture, Soil Conservation Service: Washington, DC, USA, 1972; pp. 1-127.

169. Govers, G.; Loch, R.J. Effects of initial water content and soil mechanical strength on the runoff erosion resistance of clay soils. Aust. J. Soil Res. 1993, 31, 549-566. [CrossRef]

170. Huang, C.; Wells, L.K.; Norton, L.D. Sediment transport capacity and erosion processes: Model concepts and reality. Earth Surf. Process. Landf. 1999, 24, 503-516. [CrossRef]

171. Littleboy, M.; Freebaim, D.M.; Hammer, G.L.; Silbum, D.M. Impact of soil erosion on production in cropping systems. II.* Simulation of production and erosion risks for a wheat cropping system. Aust. J. Soil Res. 1992, 30, 775-788. [CrossRef]

172. Gaiser, T.; Perkons, U.; Küpper, P.M.; Kautz, T.; Uteau-Puschmann, D.; Ewert, F.; Enders, A.; Krauss, G. Modeling biopore effects on root growth and biomass production on soils with pronounced sub-soil clay accumulation. Ecol. Modell. 2013, $256,6-15$. [CrossRef]

173. Sankey, J.B.; Sankey, T.T.; Li, J.; Ravi, S.; Wang, G.; Caster, J.; Kasprak, A. Quantifying plant-soil-nutrient dynamics in rangelands: Fusion of UAV hyperspectral-LiDAR, UAV multispectral-photogrammetry, and ground-based LiDAR-digital photography in a shrub-encroached desert grassland. Remote Sens. Environ. 2021, 253, 112223. [CrossRef]

174. Eekhout, J.P.C.; Millares-Valenzuela, A.; Martínez-Salvador, A.; García-Lorenzo, R.; Pérez-Cutillas, P.; Conesa-García, C.; de Vente, J. A process-based soil erosion model ensemble to assess model uncertainty in climate-change impact assessments. L. Degrad. Dev. 2021, 3920. [CrossRef]

175. Borrelli, P.; Robinson, D.A.; Panagos, P.; Lugato, E.; Yang, J.E.; Alewell, C.; Wuepper, D.; Montanarella, L.; Ballabio, C. Land use and climate change impacts on global soil erosion by water (2015-2070). Proc. Natl. Acad. Sci. USA 2020, 117, 21994-22001. [CrossRef] [PubMed]

176. OECD. Expert Meeting on Agri-biodiversity Indicators. In Agriculture and Biodiversity: Developing Indicators for Policy Analysis: Proceedings from an OECD Expert Meeting, Zürich, Switzerland, November 2001; OECD: Paris, France, 2003; ISBN 92-64-19920-9. 